\title{
Waves and Radiation Conditions in a Cuspidal Sharpening of Elastic Bodies
}

\author{
V.A. Kozlov ${ }^{1}$ • S.A. Nazarov Na,3 $^{2,4}$
}

Received: 5 July 2017 / Published online: 3 November 2017

(C) The Author(s) 2017. This article is published with open access at Springerlink.com

\begin{abstract}
Elastic bodies with cuspidal singularities at the surface are known to support wave processes in a finite volume which lead to absorption of elastic and acoustic oscillations (this effect is recognized in the engineering literature as Vibration Black Holes). With a simple argument we will give the complete description of the phenomenon of wave propagation towards the tip of a three-dimensional cusp and provide a formulation of the Mandelstam (energy) radiation conditions based on the calculation of the Umov-Poynting vector of energy transfer. Outside thresholds, in particular, above the lower bound of the continuous spectrum, these conditions coincide with ones due to the traditional Sommerfeld principle but also work at the threshold frequencies where the latter principle cannot indicate the direction of wave propagation. The energy radiation conditions supply the problem with a Fredholm operator of index zero so that a solution is determined up to a trapped mode with a finite elastic energy and exists provided the external loading is orthogonal to these modes. An example of a symmetric cuspidal body is presented which supports an infinite sequence of eigenfrequencies embedded into the continuous spectrum and the corresponding trapped modes with the exponential decay at the cusp tip. We also determine (unitary and symmetric) scattering matrices of two types and derive a criterion for the existence of a trapped mode with the power-low behavior near the tip.
\end{abstract}

Keywords Vibration Black Holes · Cuspidal singularity · Elastic waves · Energy radiation conditions · Trapped modes · Fredholm operator · Weighted spaces with detached asymptotics

\footnotetext{
V.A. Kozlov

vladimir.kozlov@liu.se

1 Department of Mathematics, Linköping University, 58183 Linköping, Sweden

2 Mathematics and Mechanics Faculty, Saint-Petersburg State University, Universitetskaya nab., 7/9, St. Petersburg, 199034, Russia

3 Peter the Great Saint-Petersburg State Polytechnical University, Polytechnicheskaya ul., 29, St. Petersburg, 195251, Russia

4 Institute of Problems of Mechanical Engineering RAS, V.O., Bolshoj pr., 61, St. Petersburg, 199178, Russia
} 
Mathematics Subject Classification $35 \mathrm{~B} 40 \cdot 74 \mathrm{~J} 20 \cdot 35 \mathrm{C} 20 \cdot 35 \mathrm{C} 07 \cdot 35 \mathrm{P} 05$

\section{Introduction}

\subsection{Formulation of the Problem}

Let $\Omega$ be a bounded elastic body in $\mathbb{R}^{3}$ with boundary $\Gamma=\partial \Omega$. We assume that the coordinate origin $\mathcal{O}$ belongs to $\Gamma$ and the punctured surface $\Gamma \backslash \mathcal{O}$ is smooth. By $\Gamma_{d}=\{x \in$ $\left.\partial \Pi_{d}: z=x_{3}<d\right\}$ we denote a neighborhood of a cuspidal singularity on the surface $\partial \Omega$, see Fig. 1, where

$$
\Pi_{d}=\left\{x=\left(x_{1}, x_{2}, x_{3}\right)=(y, z) \in \mathbb{R}^{2} \times \mathbb{R}: z \in(0, d), z^{-1-m} y \in \varpi\right\} .
$$

Here, $d>0, y=\left(y_{1}, y_{2}\right)=\left(x_{1}, x_{2}\right) \in \mathbb{R}^{2}, \varpi$ is a bounded two-dimensional domain with smooth boundary $\gamma=\partial \varpi$ and $m \geq 0$ is the sharpness exponent.

If $m=0$, the cusp becomes a conical point and the domain $\Omega$ is Lipschitz, therefore, the problem on harmonic in time oscillations

$$
\begin{aligned}
& -\frac{\partial \sigma_{k 1}}{\partial x_{1}}-\frac{\partial \sigma_{k 2}}{\partial x_{2}}-\frac{\partial \sigma_{k 3}}{\partial x_{3}}=\rho \omega^{2} u_{k} \quad \text { in } \Omega, \\
& n_{1} \sigma_{k 1}+n_{2} \sigma_{k 2}+n_{3} \sigma_{k 3}=0 \quad \text { on } \Gamma \backslash \mathcal{O}, \quad k=1,2,3,2,3,
\end{aligned}
$$

has the discrete spectrum, namely the unbounded sequence of eigenfrequencies

$$
0=\omega_{1}=\cdots=\omega_{6}<\omega_{7} \leq \omega_{8} \leq \cdots \leq \omega_{n} \leq \cdots .
$$

Here, $\sigma=\left(\sigma_{k j}\right)$ and $u=\left(u_{k}\right)$ are the stress tensor and the displacement vector, respectively, $\rho>0$ is the material density, and $n=\left(n_{1}, n_{2}, n_{2}\right)$ is the unit outward normal vector. It is known (see, e.g., [1]) that a similar structure of the spectrum is preserved for any $m \in(0,1)$. However, for $m \geq 1$, the above problem gets the continuous spectrum $\wp_{c o} \neq \varnothing$ provoking wave processes in the finite volume (1.1).

In the most interesting practical case $m=1$ such processes were observed experimentally and an engineering theory of spiked beams was suggested in [2, 3] and [4, 5] and other publications. This theory was used for constructing a series of devises absorbing elastic and acoustic oscillations (Vibration Black Holes), see ${ }^{1}$ Fig. 2.

Fig. 1 Elastic solid with a cusp

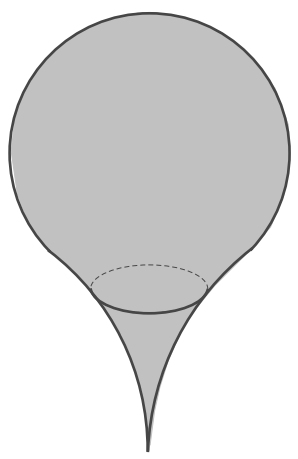

\footnotetext{
${ }^{1}$ This picture is published with permission of Professor M.A. Mironov.
} 
Fig. 2 Vibration Black Hole

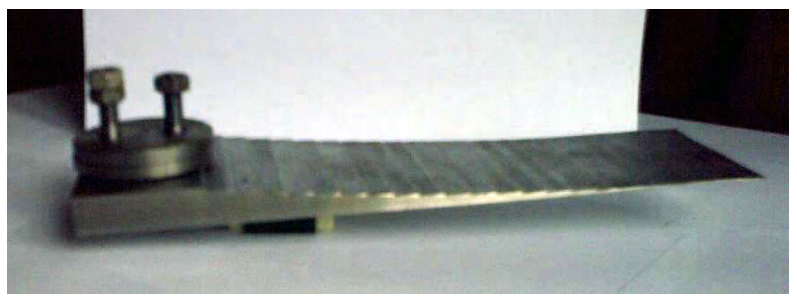

Mathematical study of the spectrum of the problem (1.2), (1.3) was started in the paper [1], where it was proved the presence of the continuous spectrum for every $m \geq 1$. In [6] it was shown that the continuous spectrum covers the whole positive axis $\overline{\mathbb{R}_{+}}=[0,+\infty)$ in the case of a "very sharp" cusp, i.e., with the sharpness exponent $m>1$. The most difficult and interesting for applications case $m=1$ was studied minutely in [7]. Based on the asymptotic methods $[8,9]$ a complete asymptotic decomposition of solutions to the problem (1.2), (1.3) was constructed and as a consequence it was proved the formula

$$
\wp_{c o}=\left[\Lambda^{\dagger}, \infty\right)
$$

for the continuous spectrum with an explicit formula, cf. (3.11) and (3.8), for the threshold $\Lambda^{\dagger}>0$. Crucially simplified but formal calculations leading to (1.5) and to explicit forms of elastic waves are presented concisely in Sect. 2 of this paper.

Since in the case

$$
\Lambda=\rho \omega^{2} \geq \Lambda^{\dagger}
$$

the operator of the elasticity problem for the elastic body $\Omega$ corresponding to the weak formulation in the Sobolev space $H^{1}(\Omega)^{3}$, loses the Fredholm property according to [7], the question about radiation condition at the tip $\mathcal{O}$ arises naturally and our paper proposes an answer to this question as well as provides a Fredholm operator to the elasticity problem and gives examples of trapped elastic modes.

\subsection{The Mandel-Voigt Notation}

In the fixed Cartesian coordinate system $x=(y, z)$ we interpret the displacement vector as a column vector $\left(u_{1}, u_{2}, u_{3}\right)^{\top}$ in $\mathbb{R}^{3}$. Here $\top$ stands for transposition and $u_{j}$ is the projection of the vector $u$ on the $x_{j}$-axis. We also introduce the strain column vector of height 6

$$
\varepsilon(u)=\left(\varepsilon_{11}(u), \varepsilon_{22}(u), \sqrt{2} \varepsilon_{12}(u), \sqrt{2} \varepsilon_{13}(u), \sqrt{2} \varepsilon_{23}(u), \varepsilon_{33}(u)\right)^{\top},
$$

where

$$
\varepsilon_{j k}=\frac{1}{2}\left(\frac{\partial u_{j}}{\partial x_{k}}+\frac{\partial u_{k}}{\partial x_{j}}\right)
$$

are Cartesian components of the strain tensor of rank 2 and the factor $\sqrt{2}$ is introduced in (1.7) to equalize the intrinsic norms of the tensor and column vector. The stress column vector has a structure similar to (1.7) and is connected with the strain column vector by Hooke's law

$$
\sigma(u)=A \varepsilon(u)
$$


where $A$ is a symmetric and positive definite $6 \times 6$-matrix composed of the material elastic moduli. To simplify our demonstration, we assume that the elastic body $\Omega$ is homogeneous but anisotropic so that the matrix $A$ and the density $\rho$ are constant. The spectral parameter $\Lambda$ is then defined according to (1.6) and throughout the paper we relate with $\Lambda$ the notation $\wp$ and $\wp_{d i}, \wp_{c o}$ for the spectrum and its discrete and continuous parts, respectively.

We note that

$$
\varepsilon(u)=D(\nabla) u,
$$

where $\nabla$ is the gradient operator and $D(\nabla)$ is $6 \times 3$-matrix of first order differential operators, namely

$$
D(\nabla)^{\top}=\left(\begin{array}{cccccc}
\partial_{1} & 0 & 2^{-1 / 2} \partial_{2} & 2^{-1 / 2} \partial_{3} & 0 & 0 \\
0 & \partial_{2} & 2^{-1 / 2} \partial_{1} & 0 & 2^{-1 / 2} \partial_{3} & 0 \\
0 & 0 & 0 & 2^{-1 / 2} \partial_{1} & 2^{-1 / 2} \partial_{2} & \partial_{3}
\end{array}\right), \quad \partial_{j}=\frac{\partial}{\partial x_{j}}
$$

The same differential operator is met in the matrix form of problem (1.2), (1.3):

$$
\begin{aligned}
& D(-\nabla)^{\top} A D(\nabla) u(x)=\Lambda u(x), \quad x \in \Omega, \\
& D(n(x))^{\top} A D(\nabla) u(x)=0, \quad x \in \Gamma \backslash \mathcal{O} .
\end{aligned}
$$

The left factor in (1.11) is obtained by the substitution $\nabla \mapsto n(x)$ in (1.9).

\subsection{Energy Space and Korn's Inequality}

The variational formulation of the problem (1.10), (1.11) runs as follows: to find non-trivial vector function $u \in H^{1}(\Omega)^{3}$ and a number $\Lambda$ satisfying the integral identity

$$
a(u, v ; \Omega)=\Lambda(u, v)_{\Omega} \quad \forall v \in H^{1}(\Omega)^{3} .
$$

Here, $(\cdot, \cdot)_{\Omega}$ is the natural inner product in the Lebesgue space $L^{2}(\Omega)$ or its vector version, $H^{1}(\Omega)$ is Sobolev space with the standard norm and the quadratic form

$$
a(u, u ; \Omega)=(A D(\nabla) u, D(\nabla) u)_{\Omega}
$$

represents the doubled elastic energy stored in the solid $\Omega$. The superscript 3 in (1.12) indicates the number of components in the test vector function $v=\left(v_{1}, v_{2}, v_{3}\right)^{\top}$ but such indexes are omitted in the notation of norms and scalar products.

If the domain $\Omega$ is Lipschitz, the Korn inequality, see, e.g., [36],

$$
\left\|u ; H^{1}(\Omega)\right\| \leq C_{\Omega}\left(\left\|D(\nabla) u ; L^{2}(\Omega)\right\|+\left\|u ; L^{2}(\Omega)\right\|\right)
$$

is valid which implies that, first, the energy space $\mathcal{W}(\Omega)$ with the natural norm

$$
\|u ; \mathcal{W}(\Omega)\|=\left(\left\|D(\nabla) u ; L^{2}(\Omega)\right\|^{2}+\left\|u ; L^{2}(\Omega)\right\|^{2}\right)^{1 / 2}
$$

coincides with the Sobolev space $H^{1}(\Omega)^{3}$ and, second, due to compactness of the embed$\operatorname{ding} H^{1}(\Omega) \subset L^{2}(\Omega)$ the spectrum of the problem (1.10), (1.11) (or equivalently (1.12)) is discrete and assembles the monotone unbounded nonnegative sequence (1.4). 
In the domain $\Omega$ with a cusp the inequality (1.14) is no longer valid and its substitute, namely a weighted anisotropic Korn inequality, was derived in [1, 10]. Its simplified version gets the form

$$
\left\|r^{m} \nabla u ; L^{2}(\Omega)\right\|+\left\|r^{-1} u_{3} ; L^{2}(\Omega)\right\|+\left\|r^{m-1} u^{\prime} ; L^{2}(\Omega)\right\| \leq C_{\Omega}\|u ; \mathcal{W}(\Omega)\| .
$$

Here, $r=\operatorname{dist}(x, \mathcal{O})$ and $u^{\prime}=\left(u_{1}, u_{2}\right)$. The distribution of weights on the left-hand side of (1.16) is optimal and the presence of vanishing (as $r \rightarrow+0$ ) weights leads to the following conclusions. First, if $m>0$, the energy space $\mathcal{W}(\Omega)$ is much larger than the Sobolev space $H^{1}(\Omega)^{3}$, in particular, the integral identity (1.12) requires a modification. Second, if $m \geq 1$, then the inclusion $\mathcal{W}(\Omega) \subset L^{2}(\Omega)^{3}$ loses its compactness and hence the spectrum of the problem (1.10), (1.11) is no longer discrete. This is one of the reasons for our study in [7] of the asymptotic behavior of elastic fields near the cusp tip and the next step is to establish the radiation conditions at the point $\mathcal{O}$.

\subsection{Structure of the Paper}

Formal asymptotic expansions of solutions to the problem (1.10), (1.11) are derived in Sect. 2 while the dimension reduction procedure leads to the limit system of ordinary differential equations of Euler's type in the longitudinal coordinate $z$. These expansions have been justified in the paper [7] but Theorem 1 in Sect. 3 provides a reformulated result which crucially simplifies all further considerations, namely we prove that the formal asymptotic expansion gives rise to certain three-dimensional elastic fields which entirely satisfy the problem (1.10), (1.11) in the $d^{\prime}$-neighborhood of the cusp tip $\mathcal{O}$ with some $d^{\prime} \in(0, d)$ and have power-law solutions of the limit system as the main asymptotic terms. It is important that any solution of the whole problem with a slow exponential growth can be represented as a linear combination of these solutions and a remainder which decays exponentially as $x \rightarrow \mathcal{O}$.

Furthermore, in Sect. 3 we classify the above-mentioned solutions and pay the most attention to those who have the singularities $z^{ \pm i \kappa-5 / 2}$ with certain $\kappa \in \mathbb{R}$, are called oscillatory waves, and transport elastic energy along the cusp (1.1). Based on the Mandelstam radiation conditions, we moreover classify the waves as incoming from and outgoing to the tip $\mathcal{O}$.

In Sect. 4 we proceed with computing the Umov-Poynting vector of the elastic energy transfer which implies the symplectic form $q$, see (4.4), indicates the direction of wave propagation and supports the above-mentioned Mandelstam classification. Moreover, the form $q$ allows us to normalize the incoming and outgoing waves, cf., Sect. 4.3, and, as a result, to introduce the unitary and symmetric scattering matrix $S$. In a similar way, we introduce the packets of the energy $(\kappa>0)$ and non-energy $(\kappa<0)$ waves with the singularities $z^{\kappa-5 / 2}$ which involve a real exponent $\kappa$ and make the total energy functional finite and infinite, respectively. These packets generate the augmented scattering matrix $\mathfrak{S}$. It is an artificial object but imitates the classical scattering matrix, inherits its natural unitary and symmetry properties, and plays the most important role in our description of elastic trapped modes in the solid $\Omega$.

Section 5 is devoted to examination of the solvability of the problem

$$
\begin{aligned}
& D(-\nabla)^{\top} A D(\nabla) u(x)-\Lambda u(x)=f(x), \quad x \in \Omega, \\
& D(n(x))^{\top} A D(\nabla) u(x)=g(x), \quad x \in \Gamma \backslash \mathcal{O},
\end{aligned}
$$


cf., the homogeneous problem (1.10), (1.11), under certain assumptions on the right-hand sides $f$ and $g$. First of all, we verify in Sect. 5.1 that, for

$$
\Lambda \in\left[0, \Lambda^{\dagger}\right)
$$

i.e., below the threshold $\Lambda^{\dagger}>0$, see (1.5), the variational formulation of the elasticity problem in the cuspidal domain $\Omega$ readily generates a Fredholm operator in the energy space $\mathcal{W}(\Omega)$ (Proposition 1). In other words, under the restriction (1.19), the cuspidal boundary irregularity (1.1) does not change the general properties of the elasticity problem.

In Sect. 5.2 we prove that in the rotational symmetric isotropic bodies the problem (1.10), (1.11) have an infinite unbounded sequence of eigenvalues while the corresponding elastic eigenmodes enjoy the exponential decay as $x \rightarrow \mathcal{O}$. It is remarkable that an infinite part of this sequence belongs to the continuous spectrum (1.5), i.e., we present an example of an unbounded family of embedded eigenvalues.

In Sect. 5.3 we determine the augmented scattering matrix $\mathfrak{S}$ and verify its properties. It gives in Theorem 2 a criterion of the existence of trapped modes with the power-law behavior near the tip $\mathcal{O}$. Moreover, a simple formula (5.41) reproduces the traditional scattering matrix $S$ which describes the wave processes in the elastic body $\Omega$. Finally, in Sect. 5.5 we formulate the (energy) Mandelstam radiation conditions and provide a Fredholm operator of index zero for the problem (1.17), (1.18) in weighted spaces with detached asymptotics (Theorem 3).

It is worth mentioning that the detailed investigation in Sects. 2-4 of asymptotics inside the cusp allows us to reduce the mathematical tools in Sect. 5 to quite simple algebraic operations and does not require a complicated analysis of the original boundary value problem.

\section{Formal Asymptotic Analysis}

\subsection{Preamble}

The cusp (1.1) has a special geometrical feature, namely the diameter $O\left(z_{0}^{1+m}\right)$ of the crosssection

$$
\varpi\left(z_{0}\right)=\left\{x \in \Pi_{d}: z=z_{0}\right\}
$$

is much smaller that the distance $z_{0}>0$ from $\varpi\left(z_{0}\right)$ to the cusp tip $\mathcal{O}$. This observation suggests to apply the theory of thin elastic rods (see [11,12] for engineering theories and [13], [14, Ch. 6], [15, Ch. 5], [16] for mathematical results). In this section we use the asymptotic procedure of the dimension reduction [15, 17], see also [1, 18-20] for application to elastic cusps, in order to derive a limit system of ordinary differential equations in the variable $z$. However, this approach cannot justify asymptotic expansions. For an elastic rod, e.g., with a constant cross-section, estimates for asymptotic remainders are usually derived by means of variants of Korn's inequality (cf., the review paper [21]) but the weighted Korn inequality (1.16) cannot help to achieve the goal mainly due to the degeneration of the cross-section $\varpi(z)$ as $z \rightarrow+0$. In order to formulate radiation conditions and to present the Fredholm operator of the problem we make use of results in [7] which are based on a different, much more elaborated technique $[8,9]$, where such problems are treated as an abstract ordinary differential equation with variable operator coefficients and an asymptotic theory based on a spectral splitting, is developed in the above cited references. 


\subsection{Asymptotic Ansatz}

In what follows we always consider the sharpness exponent $m=1$ which is accepted in all engineering experiments and applicable works. In the mathematical theory of thin elastic rods with variable cross-section (compare formulas $(2.1),(1.9)$ and, cf., $[15,17,22])$ the displacement vector is sought in the form

$$
u(x)=U^{-2}(y, z)+U^{-1}(y, z)+U^{0}(y, z)+U^{1}(y, z)+U^{2}(y, z)+\cdots,
$$

where the first pair of terms is independent of the elastic properties of the body $\Omega$ and the shape of the cross-section $\varpi$, namely

$$
\begin{aligned}
& U^{-2}(y, z)=\sum_{j=1}^{2} w_{j}(z) e_{(j)}, \\
& U^{-1}(y, z)=\left(w_{3}(z)-\sum_{j=1}^{2} y_{j} \frac{\partial w_{j}}{\partial z}(z)\right) e_{(3)}+w_{4}(z) \theta(y) .
\end{aligned}
$$

Here, $e_{(k)}$ is the unit vector of the $x_{k}$-axis and the linear vector function

$$
\theta(y)=2^{-1 / 2}\left(y_{1} e_{(2)}-y_{2} e_{(1)}\right)
$$

corresponds to rotation about the axis of the cusp. Notice that the additional factor $2^{1 / 2}$ in comparison with the usual definition of the rotation vector is in consistence with the previous formulas (1.7) and (1.9) containing these factors, too. The functions $w_{1}, w_{2}, w_{3}$ and $w_{4}$ in (2.3) and (2.4) assemble the vector function

$$
w=\left(w_{1}, w_{2}, w_{3}, w_{4}\right)^{\top},
$$

which will be found later on together with the higher-order terms $U^{0}, U^{1}, \ldots$ in the asymptotic ansatz (2.2).

Since the main goal of our paper is to describe the behavior of elastic fields near the cusp tip $\mathcal{O}$, i.e., as $z \rightarrow+0$, we have to specify the dependence on $z$ of each term in the ansatz (2.2). We note that the first term (2.3) generates bending of the cusp (1.1) and the sum $w_{3}(z) e_{(3)}+w_{4}(z) \theta(y)$ in (2.4) corresponds to the longitudinal displacement and twisting of $\Pi_{d}$. Referring to the experience of everyday life, we recall that to bend any rod is much easier than to stretch or twist it. Therefore, we assign a faster growth as $z \rightarrow+0$ to the summand (2.3) than to other summands in (2.2) as follows:

$$
\begin{aligned}
U^{-2}(z) & =z^{\kappa-5 / 2} \mathcal{U}^{-2}, \\
U^{p}(y, z) & =z^{p+2+\kappa-5 / 2} \mathcal{U}^{-p}(\eta), \quad p=-1,0,1 .
\end{aligned}
$$

Here, $\kappa \in \mathbb{C}, z^{-5 / 2}$ is a convenient normalization factor, $\mathcal{U}^{-2}$ is a constant column vector and $\mathcal{U}^{-1}, \mathcal{U}^{0}, \mathcal{U}^{1}$ are vector functions in the fast transversal variables

$$
\eta=\left(\eta_{1}, \eta_{2}\right)=z^{-2} y
$$

The variables (2.9) are introduced in such a way that the cross-section of the cusp is given by the simple inclusion $\eta \in \varpi$. 
According to the basic formulas (2.3) and (2.4), the relations (2.7) as well as (2.8) with $p=-1$ are satisfied provided

$$
\begin{aligned}
& w_{j}(z)=z^{\kappa-5 / 2} \mathcal{W}_{j}, \quad j=1,2, \\
& w_{3}(z)=z^{\kappa-3 / 2} \mathcal{W}_{3}, \quad w_{4}(z)=z^{\kappa-7 / 2} \mathcal{W}_{4},
\end{aligned}
$$

where $\mathcal{W}=\left(\mathcal{W}_{1}, \mathcal{W}_{2}, \mathcal{W}_{3}, \mathcal{W}_{4}\right)^{\top}$ is a constant column vector. It is important that, by (2.9) and (2.5), we obtain

$$
w_{4}(z) \theta(y)=z^{\kappa-3 / 2} \mathcal{W}_{4} \theta(\eta)
$$

Validation of the accepted anzätze is confirmed by results in [1, 7] and our consideration below.

\subsection{Splitting Differential Operators}

Due to the definition (1.1) the normal vector at the surface $\Gamma_{d}=\{x \in \partial \Omega: z<d\}=\{x \in$ $\left.\partial \Pi_{d}: z<d\right\}$ takes the form

$$
n(y, z)=n_{0}(\eta, z)^{-1}\left(v_{1}(\eta), v_{2}(\eta),-2 z \eta^{\top} v(\eta)\right)^{\top}, \quad n_{0}(\eta, z)=\left(1+4 z^{2}\left|\eta^{\top} v(\eta)\right|^{2}\right)^{1 / 2},
$$

where $\eta=\left(\eta_{1}, \eta_{2}\right)^{\top}$ is a column vector of the stretched coordinates (2.9), $v=\left(v_{1}, v_{2}\right)^{\top}$ is the unit outward normal to the boundary $\gamma$ of the domain $\varpi$ in the plane $\mathbb{R}^{2}$ and $n_{0}$ is a normalization factor.

The matrix differential operators $L(\nabla)=L\left(\nabla_{y}, \partial_{z}\right)$ and $N(x, \nabla)=N\left(y, z, \nabla_{y}, \partial_{z}\right)$ on the left-hand sides of Eqs. (1.10) and (1.11), respectively, admit the decompositions

$$
L=L^{0}+L^{1}+L^{2}, \quad n_{0} N=N^{0}+N^{1}+N^{2},
$$

where $^{2}$

$$
\begin{aligned}
& L^{0}\left(\nabla_{y}\right)=D\left(-\nabla_{y}, 0\right)^{\top} A D\left(\nabla_{y}, 0\right), \\
& L^{1}\left(\nabla_{y}, \partial_{z}\right)=D\left(-\nabla_{y}, 0\right)^{\top} A D\left(0, \partial_{z}\right)+D\left(0,-\partial_{z}\right)^{\top} A D\left(\nabla_{y}, 0\right), \\
& L^{2}\left(\partial_{z}\right)=D\left(0,-\partial_{z}\right)^{\top} A D\left(0, \partial_{z}\right), \\
& N^{0}\left(y, z, \nabla_{y}\right)=D\left(v\left(z^{-2} y\right), 0\right)^{\top} A D\left(\nabla_{y}, 0\right), \\
& N^{1}\left(y, z, \nabla_{y}, \partial_{z}\right)=D\left(v\left(z^{-2} y\right), 0\right)^{\top} A D\left(0, \partial_{z}\right)-2 z D\left(0, z^{-2} y^{\top} v\left(z^{-2} y\right)\right)^{\top} A D\left(\nabla_{y}, 0\right), \\
& N^{2}\left(y, z, \partial_{z}\right)=-2 z D\left(0, z^{-2} y^{\top} v\left(z^{-2} y\right)\right)^{\top} A D\left(0, \partial_{z}\right)
\end{aligned}
$$

and $\partial_{z}=\partial / \partial z, \nabla_{y}=\left(\partial / \partial y_{1}, \partial / \partial y_{2}\right)^{\top}$. We note that

$$
\begin{aligned}
& \nabla_{y}\left(z^{\kappa} \mathcal{V}\left(z^{-2} y\right)\right)=\left.z^{\kappa-2} \nabla_{\eta} \mathcal{V}(\eta)\right|_{\eta=z^{-2} y} \\
& \partial_{z}\left(z^{\kappa} \mathcal{V}\left(z^{-2} y\right)\right)=\left.z^{\kappa-1}\left(\kappa \mathcal{V}(\eta)-2 \eta^{\top} \nabla_{\eta} \mathcal{V}(\eta)\right)\right|_{\eta=z^{-2} y}
\end{aligned}
$$

\footnotetext{
${ }^{2}$ Although the symbols $L^{2}\left(\partial_{z}\right)$ and $L^{2}(\Omega)$ of the introduced differential operator and the Lebesgue space look quite similar, no misunderstanding may occur in what follows.
} 
i.e., the gradient operator $\nabla_{y}$ in the transversal variables reduces the exponent by two but the derivative $\partial_{z}$ in the longitudinal variable by one only. Therefore, the operators $L^{p}$ and $N^{p}$ from (2.14) are formally assigned with conventional orders $p-4$ and $p-2$, respectively.

Inserting (2.2) and (2.13) into Eqs. (1.10) and (1.11) restricted onto the sets $\Pi_{d}$ and $\Gamma_{d} \backslash \mathcal{O}$, respectively, we collect terms of alike orders in $z$ and compose two-dimensional elastic problems on the cross-section $\varpi(z)$ of the cusp $\Pi_{d}$, see (2.1) and (1.1),

$$
\begin{aligned}
& L^{0} U^{q}=F^{q}:=-L^{1} U^{q-1}-L^{2} U^{q-2}+\Lambda \delta_{2, q} U^{-2} \quad \text { in } \varpi(z), \\
& N^{0} U^{q}=G^{q}:=-N^{1} U^{q-1}-N^{2} U^{q-2} \quad \text { on } \partial \varpi(z)=: \gamma(z) .
\end{aligned}
$$

Here, $q=-2, \ldots, 2, U^{p}=0$ for $p<-2$, and $\delta_{p, q}$ is the Kronecker symbol. We emphasize that the inertial term $\Lambda u$ on the right-hand side of (1.10) generates the term $\Lambda U^{-2}$ in the problem (2.15) for $U^{2}$.

\subsection{Solving the Recurrent Sequence of Problems}

As was shown in [1], see also [7, 17, 22] and [15, Sect. 7.3], the sequence of the problems (2.15) and their compatibility conditions lead to one-dimensional limit spectral ordinary differential equations in the variable $z$. Let us implement this procedure.

First of all, we observe that the vector functions (2.3) and (2.4) satisfy the problems (2.15) with $q=-2$ and $q=-1$, respectively. Indeed, $F^{-2}=0, G^{-2}=0$ and the operators $L^{0}$ and $N^{0}$ differentiate in the variables $y=\left(y_{1}, y_{2}\right)$ only but the latter are absent in $U^{-2}$. Furthermore, according to (1.9), we have

$$
D\left(\nabla_{y}, 0\right) y_{j} e_{(3)}=D\left(e_{(3)}\right) e_{(j)}, \quad j=1,2 .
$$

Using these equalities together with formulas (2.14) for $L^{0}, N^{0}$ and $L^{1}, N^{1}$ as well as the definition (2.3), (2.4) of $U^{-2}, U^{-1}$, one checks that relations (2.15) with $q=-1$ are satisfied too.

The rigid motions $e_{(1)}, e_{(2)}, e_{(3)}$ (translations) and $\theta(y)$ (rotation) form a basis in the subspace of solutions to the homogeneous (cf. (2.15) with $F^{q}=0$ and $G^{q}=0$ ) elasticity problem in $\varpi(z) \subset \mathbb{R}^{2}$ : indeed, the formulas

$$
D\left(\nabla_{y}, 0\right) e_{(k)}=0, \quad k=1,2,3, \quad D\left(\nabla_{y}, 0\right) \theta(y)=0
$$

are evident and, moreover,

$$
D\left(\nabla_{y}, 0\right) v(y)=0 \quad \Leftrightarrow \quad v(y)=\left(a_{1}-a_{4} y_{2}, a_{2}+a_{4} y_{1}, a_{3}\right)^{\top} .
$$

Thus, according to the Fredholm alternative, the problem (2.15) is solvable if and only if the following four compatibility conditions are valid:

$$
\begin{aligned}
& \int_{\varpi(z)} F_{k}^{q}(y, z) d y+\int_{\partial \varpi(z)} G_{k}^{q}(y, z) d s_{y}=0, \quad k=1,2,3, \\
& \int_{\varpi(z)} \theta(y)^{\top} F^{q}(y, z) d y+\int_{\partial \varpi(z)} \theta(y)^{\top} G^{q}(y, z) d s_{y}=0,
\end{aligned}
$$

where $F_{k}^{q}$ and $G_{k}^{q}$ are Cartesian components of the vectors $F^{q}$ and $G^{q}$ respectively. 
The right-hand sides of the problem (2.15) with $q=0$ take the form

$$
\begin{aligned}
F^{0} & =-L^{1} U^{-1}-L^{2} U^{-2} \\
& =D\left(\nabla_{y}, 0\right)^{\top} A D\left(0, \partial_{z}\right) U^{-1}+D\left(0, \partial_{z}\right)^{\top} A\left(D\left(\nabla_{y}, 0\right) U^{-1}+D\left(0, \partial_{z}\right) U^{-2}\right), \\
G^{0} & =-N^{1} U^{-1}-N^{2} U^{-2} \\
& =-D(v, 0)^{\top} A D\left(0, \partial_{z}\right) U^{-1}+2 z D\left(0, z^{-2} y^{\top} v\right)^{\top} A\left(D\left(\nabla_{y}, 0\right) U^{-1}+D\left(0, \partial_{z}\right) U^{-2}\right) .
\end{aligned}
$$

Since $D\left(\nabla_{y}, 0\right) U^{-1}+D\left(0, \partial_{z}\right) U^{-2}=0$, the last terms on the right-hand sides of (2.18) vanish due to (2.3), (2.4) and (1.9). Therefore,

$$
F^{0}=D\left(\nabla_{y}, 0\right)^{\top} A Y \mathcal{D}\left(\partial_{z}\right) w, \quad G^{0}=D(v, 0)^{\top} A Y \mathcal{D}\left(\partial_{z}\right) w
$$

where

$$
Y(y)=\left(\begin{array}{cccccc}
0 & 0 & 0 & 0 & 0 & -y_{1} \\
0 & 0 & 0 & 0 & 0 & -y_{2} \\
0 & 0 & 0 & 0 & 0 & 1 \\
0 & 0 & 0 & -\frac{1}{2} y_{2} & \frac{1}{2} y_{1} & 0
\end{array}\right)^{\top}, \quad \mathcal{D}\left(\partial_{z}\right)=\left(\begin{array}{cccc}
\partial_{z}^{2} & 0 & 0 & 0 \\
0 & \partial_{z}^{2} & 0 & 0 \\
0 & 0 & \partial_{z} & 0 \\
0 & 0 & 0 & \partial_{z}
\end{array}\right) .
$$

Integrating by parts, one easily verifies the compatibility conditions (2.17) with $q=0$ and the representation

$$
U^{0}(y, z)=X(y, z) \mathcal{D}\left(\partial_{z}\right) w(z),
$$

where $X=\left(X^{1}, X^{2}, X^{3}, X^{4}\right)$ is a $3 \times 4$-matrix function satisfying the problem

$$
\begin{aligned}
& D\left(-\nabla_{y}, 0\right)^{\top} A D\left(\nabla_{y}, 0\right) X(y, z)=D\left(\nabla_{y}, 0\right)^{\top} A Y(y), \quad y \in \varpi(z), \\
& D\left(v\left(z^{-2} y, 0\right)^{\top} A D\left(\nabla_{y}, 0\right) X(y, z)=D\left(v\left(z^{-2} y, 0\right)^{\top} A Y(y), \quad y \in \partial \varpi(z) .\right.\right.
\end{aligned}
$$

Formulas (2.22) accept $3 \times 4$-matrices, i.e., the column vectors $X^{1}, \ldots, X^{4}$ are solutions of the two-dimensional elasticity problem with special right-hand sides. The structure of the matrix $Y$ in (2.20) guarantees the following relations:

$$
X^{l}(y, z)=z^{4} \mathcal{X}^{l}\left(z^{-2} y\right), \quad l=1,2,4, \quad X^{3}(y, z)=z^{2} \mathcal{X}^{3}\left(z^{-2} y\right) .
$$

We now consider the problem (2.15) with $q=1$ which gets the right-hand sides

$$
\begin{aligned}
F^{1} & =D\left(\nabla_{y}, 0\right)^{\top} A D\left(0, \partial_{z}\right) U^{0}+D\left(0, \partial_{z}\right) A\left(D\left(\nabla_{y}, 0\right) U^{0}+D\left(0, \partial_{z}\right) U^{-1}\right) \\
& =D\left(\nabla_{y}, 0\right)^{\top} A D\left(0, \partial_{z}\right) U^{0}+D\left(0, \partial_{z}\right)^{\top} A\left(D\left(\nabla_{y}, 0\right) X+Y\right) \mathcal{D}\left(\partial_{z}\right) w, \\
G^{1} & =-D(v, 0)^{\top} A D\left(0, \partial_{z}\right) U^{0}+2 z D\left(0, z^{-2} y^{\top} v\right)^{\top} A\left(D\left(\nabla_{y}, 0\right) X+Y\right) \mathcal{D}\left(\partial_{z}\right) w .
\end{aligned}
$$

Notice that, according to the definitions (1.1) and (2.1), any smooth function $T$ satisfies the relation

$$
\frac{d}{d z} \int_{\varpi(z)} T(y, z) d y=\int_{\varpi(z)} \frac{\partial T}{\partial z}(y, z) d y+2 z \int_{\partial \varpi(z)}\left(z^{-2} y\right)^{\top} v\left(z^{-2} y\right) T(y, z) d s_{y} .
$$

Let us proceed with the first two $(k=1,2)$ compatibility conditions in (2.17). The first summand on the right-hand side of (2.24) does not contribute in these conditions due 
to Gauss-Ostrogradsky Theorem. Using the formula (2.25) with $T=D(0,1)^{\top} A\left(D\left(\nabla_{y}\right.\right.$, 0) $\left.U^{0}+D\left(0, \nabla_{z}\right) U^{-1}\right)$, we derive

$$
\begin{aligned}
\int_{\varpi(z)} & F_{k}^{1} d y+\int_{\partial \varpi(z)} G_{k}^{1} d s_{y} \\
= & \frac{d}{d z} e_{(k)}^{\top} \int_{\varpi(z)} D\left(e_{(3)}\right)^{\top} A\left(D\left(\nabla_{y}, 0\right) X+Y\right) d y \mathcal{D}\left(\partial_{z}\right) w \\
= & \frac{d}{d z} \int_{\varpi(z)}\left(D\left(\nabla_{y}, 0\right) y_{k} e_{(3)}\right)^{\top} A\left(D\left(\nabla_{y}, 0\right) X+Y\right) d y \mathcal{D}\left(\partial_{z}\right) w \\
= & \frac{d}{d z} e_{(3)}\left(\int_{\varpi(z)} y_{k} D\left(-\nabla_{y}, 0\right)^{\top} A\left(D\left(\nabla_{y}, 0\right) X+Y\right) d y\right. \\
& \left.+\int_{\partial \varpi(z)} y_{k} D(v, 0)^{\top} A\left(D\left(\nabla_{y}, 0\right) X+Y\right) d s_{y}\right) \mathcal{D}\left(\partial_{z}\right) w, \quad k=1,2 .
\end{aligned}
$$

Here, we have applied the identity (2.16). Now the right-hand side of (2.26) vanishes because of Eq. (2.22).

Dealing with two other compatibility conditions involving the vectors $e_{(3)}$ and $\theta$, we proceed in a similar way, namely the formula (2.25) yields

$$
\begin{aligned}
-\frac{\partial}{\partial z} \int_{\varpi(z)} e_{(3)}^{\top} D\left(e_{(3)}\right)^{\top} A\left(D\left(\nabla_{y}, 0\right) X(y, z)+Y(y)\right) d y \mathcal{D}\left(\partial_{z}\right) w & =0, \\
-\frac{\partial}{\partial z} \int_{\varpi(z)} \theta(y)^{\top} D\left(e_{(3)}\right)^{\top} A\left(D\left(\nabla_{y}, 0\right) X(y, z)+Y(y)\right) d y \mathcal{D}\left(\partial_{z}\right) w & =0 .
\end{aligned}
$$

Finally, we consider two first $(k=1,2)$ compatibility conditions $(2.17)$ with $q=2$. The corresponding problem (2.15) involves the vector functions

$$
\begin{aligned}
& F^{2}=D\left(\nabla_{y}, 0\right)^{\top} A D\left(0, \partial_{z}\right) U^{1}+D\left(0, \partial_{z}\right)^{\top} A\left(D\left(\nabla_{y}, 0\right) U^{1}+D\left(0, \partial_{z}\right) U^{0}\right)+\Lambda U^{-2}, \\
& G^{2}=-D(v, 0)^{\top} A D\left(0, \partial_{z}\right) U^{1}+2 z D\left(0, z^{-2} y^{\top} v\right)^{\top} A\left(D\left(\nabla_{y}, 0\right) U^{1}+D\left(0, \partial_{z}\right) U^{0}\right) .
\end{aligned}
$$

Similarly to (2.24), the first terms on the right-hand sides cancel each other after integrating over $\varpi(z)$ and $\partial \varpi(z)$, respectively. The remaining terms are converted as in (2.26) and we obtain

$$
\begin{aligned}
\Lambda z^{4}|\varpi| w_{k}= & \frac{d}{d z} e_{(k)}^{\top} \int_{\varpi(z)} D\left(e_{(3)}\right)^{\top} A\left(D\left(\nabla_{y}, 0\right) U^{1}+D\left(0, \partial_{z}\right) U^{0}\right) d y \mathcal{D}\left(\partial_{z}\right) w \\
= & \frac{d}{d z} e_{(k)}^{\top} \int_{\varpi(z)}\left(D\left(\nabla_{y}\right) y_{k}\right)^{\top} A\left(D\left(\nabla_{y}, 0\right) U^{1}+D\left(0, \partial_{z}\right) U^{0}\right) d y \mathcal{D}\left(\partial_{z}\right) w \\
= & -\frac{d}{d z} e_{(k)}^{\top}\left(\int_{\varpi(z)} y_{k} D\left(\nabla_{y}, 0\right)^{\top} A\left(D\left(\nabla_{y}, 0\right) U^{1}+D\left(0, \partial_{z}\right) U^{0}\right) d y\right. \\
& \left.-\int_{\partial \varpi(z)} y_{k} D(v, 0)^{\top} A\left(D\left(\nabla_{y}, 0\right) U^{1}+D\left(0, \partial_{z}\right) U^{0}\right) d s_{y}\right) \mathcal{D}\left(\partial_{z}\right) w .
\end{aligned}
$$

Note that $\left|\varpi\left(z_{0}\right)\right|=z_{0}^{4}|\varpi|$ is the area of the cross-section (2.1). Now taking the equality (2.15) with $q=1$ and the relations (2.24) into account, we transform the difference of two 
integrals in the last two lines of (2.28) into the expression

$$
\begin{aligned}
& -\int_{\varpi(z)} y_{k} D\left(0, \partial_{z}\right)^{\top} A\left(D\left(\nabla_{y}, 0\right) U^{0}+D\left(0, \partial_{z}\right) U^{-1}\right) d y \\
& \quad-2 z^{2} \int_{\partial \varpi(z)} y_{k} D\left(0, z^{-2} y^{\top} v\right)^{\top} A\left(D\left(\nabla_{y}, 0\right) U^{0}+D\left(0, \partial_{z}\right) U^{-1}\right) d s_{y} \\
& =-\frac{d}{d z} D\left(e_{(3)}\right)^{\top} \int_{\varpi(z)} y_{k} A\left(D\left(\nabla_{y}, 0\right) X+Y\right) d y \mathcal{D}\left(\partial_{z}\right) w .
\end{aligned}
$$

Here, we have used formulas (2.25), (2.21) and (2.19). As a result, we append (2.27) with two differential equations

$$
\frac{d^{2}}{d z^{2}} e_{(3)}^{\top} D\left(e_{(3)}\right)^{\top} \int_{\varpi(z)} y_{k} A\left(D\left(\nabla_{y}, 0\right) X+Y\right) d y \mathcal{D}\left(\partial_{z}\right) w=\Lambda z^{4}|\varpi| w_{k}, \quad k=1,2 .
$$

\subsection{The Limit System of Ordinary Differential Equations}

First, we note that the column vectors

$$
-D\left(e_{(3)}\right) e_{(3)} y_{1},-D\left(e_{(3)}\right) e_{(3)} y_{2} \quad \text { and } \quad D\left(e_{(3)}\right) e_{(3)}, D\left(e_{(3)}\right) \theta
$$

forming the matrix $Y(y)$ in (2.20), appear on the left-hand sides of Eqs. (2.30) and (2.27) respectively (transposed and with minus sign). Thus, the system obtained in the previous section can be rewritten in a concise, that is, matrix form

$$
\mathcal{D}\left(-\partial_{z}\right)^{\top} \mathcal{A}(z) \mathcal{D}\left(\partial_{z}\right) w(z)=\Lambda|w| z^{4} \mathcal{T} w(z), \quad z>0 .
$$

Here, $\mathcal{T}=\operatorname{diag}\{1,1,0,0\}$ is the diagonal matrix and the matrix $\mathcal{A}$ enjoys the representation

$$
\begin{aligned}
\mathcal{A}(z) & =\int_{\varpi(z)} Y(y)^{\top} A\left(D\left(\nabla_{y}, 0\right) X(y, z)+Y(y)\right) d y \\
& =\int_{\varpi(z)}\left(D\left(\nabla_{y}, 0\right) X(y, z)+Y(y)\right)^{\top} A\left(D\left(\nabla_{y}, 0\right) X(y, z)+Y(y)\right) d y .
\end{aligned}
$$

The last equality is supported by Eq. (2.22) because

$$
\begin{aligned}
\int_{\varpi(z)} & \left(D\left(\nabla_{y}, 0\right) X(y, z)\right)^{\top} A\left(D\left(\nabla_{y}, 0\right) X(y, z)+Y(y)\right) d y \\
= & -\int_{\varpi(z)} X(y, z)^{\top} D\left(\nabla_{y}, 0\right)^{\top} A\left(D\left(\nabla_{y}, 0\right) X(y, z)+Y(y)\right) d y \\
& +\int_{\partial \varpi(z)} X(y, z)^{\top} D(v, 0)^{\top} A\left(D\left(\nabla_{y}, 0\right) X(y, z)+Y(y)\right) d s_{y} .
\end{aligned}
$$

The matrix $\mathcal{A}(z)$ of size $4 \times 4$ is a Gram matrix, i.e., it is symmetric and positive definite (a proof can be found in the papers [17, 22], the book [15, Proposition 5.1.2], and others). Furthermore, the formulas (2.20) and (2.24) maintain the polynomial dependence on the variable $z$ :

$$
\mathcal{A}(z)=\operatorname{diag}\left\{z^{4}, z^{4}, z^{2}, z^{4}\right\} \mathcal{A}(1) \operatorname{diag}\left\{z^{4}, z^{4}, z^{2}, z^{4}\right\} .
$$


Therefore, (2.31) is a system of differential equations of Euler's type and admits solutions in the form (2.6), (2.10).

\subsection{Isotropic Homogeneous Elastic Material}

For an isotropic material, the matrix $A$ from Hooke's law (1.8) looks as follows:

$$
A=\left(\begin{array}{cccccc}
\lambda+2 \mu & \lambda & 0 & 0 & 0 & \lambda \\
\lambda & \lambda+2 \mu & 0 & 0 & 0 & \lambda \\
0 & 0 & 2 \mu & 0 & 0 & 0 \\
0 & 0 & 0 & 2 \mu & 0 & 0 \\
0 & 0 & 0 & 0 & 2 \mu & 0 \\
\lambda & \lambda & 0 & 0 & 0 & \lambda+2 \mu
\end{array}\right),
$$

where $\lambda \geq 0$ and $\mu>0$ are the Lamè constants. The matrix $\mathcal{A}(z)$ from (2.31) takes an explicit form (see [13], [17], [23, Sect. 3.6], [15, §5.2] etc.), namely the constant middle multiplier in the decomposition (2.33) has the representation

$$
\mathcal{A}(1)=\left(\begin{array}{cccc}
E I_{11}(\varpi) & E I_{12}(\varpi) & -E|\varpi| P_{1}(\varpi) & 0 \\
E I_{21}(\varpi) & E I_{22}(\varpi) & -E|\varpi| P_{2}(\varpi) & 0 \\
-E|\varpi| P_{1}(\varpi) & -E|\varpi| P_{2}(\varpi) & E|\varpi| & 0 \\
0 & 0 & 0 & \mu G(\varpi) / 2
\end{array}\right) .
$$

Here, $E=\mu \frac{3 \lambda+2 \mu}{\lambda+\mu}$ is Young's modulus while $|\varpi|, P(\varpi)$, and $I(\varpi)$ are the area, the center of gravity, and the inertia tensor of the domain $\varpi \in \mathbb{R}^{2}$, respectively, that is,

$$
|\varpi|=\int_{\varpi} d y, \quad P_{j}(\varpi)=\frac{1}{|\varpi|} \int_{\varpi} y_{j} d y, \quad I_{j k}=\int_{\varpi} y_{j} y_{k} d y .
$$

Furthermore, $G(\varpi)$ is the torsional stiffness of the cross-section $\varpi$, which is calculated through the Prandtl stress function $\Psi$ :

$$
\begin{aligned}
& -\Delta \Psi(y)=2, \quad y \in \varpi, \quad \Psi(y)=0, \quad y \in \partial \varpi, \\
& G(\varpi)=\int_{\varpi}\left(\left|\frac{\partial \Psi}{\partial y_{1}}(y)\right|^{2}+\left|\frac{\partial \Psi}{\partial y_{2}}(y)\right|^{2}\right) d y>0 .
\end{aligned}
$$

If the coordinate origin coincides with the center of gravity of the domain $\varpi$ and the $y_{j}$-axes, $j=1,2$, are directed along the principal axes of the inertia tensor $I(\varpi)$, we obtain

$$
P_{1}(\varpi)=P_{2}(\varpi)=0, \quad I_{12}(\varpi)=I_{21}(\varpi)=0
$$

so that the matrix $\mathcal{A}(z)$ becomes diagonal.

\section{Waves}

\subsection{Reduction of the Resulting System of Ordinary Differential Equations}

We split the matrix (2.32) into blocks of size $2 \times 2$ as follows:

$$
\mathcal{A}(z)=\left(\begin{array}{ll}
\mathcal{A}_{\sharp \sharp}(z) & \mathcal{A}_{\sharp \bullet}(z) \\
\mathcal{A}_{\bullet \sharp}(z) & \mathcal{A}_{\bullet \bullet}(z)
\end{array}\right) .
$$


In what follows we also split column vectors in $\mathbb{R}^{4}$ into a couple of column vectors of height 2 and supply the latter with subscripts $\sharp$ and $\bullet$. In this way we introduce the vector functions

$$
w_{\sharp}=\left(w_{1}, w_{2}\right)^{\top}, \quad w_{\bullet}=\left(w_{3}, w_{4}\right)^{\top} .
$$

In the new notation the last two lines (2.27) in the system (2.31) become

$$
-\partial_{z} \mathcal{A}_{\bullet}(z) \partial_{z}^{2} w_{\sharp}(z)-\partial_{z} \mathcal{A}_{\bullet \bullet}(z) \partial_{z} w_{\bullet}(z)=0, \quad z>0 .
$$

Hence, the first two lines (2.30) of the same system can be rewritten as

$$
\partial_{z}^{2} \mathcal{M}(z) \partial_{z}^{2} w_{\sharp}(z)=\Lambda z^{4}|\varpi| w_{\sharp}(z), \quad z>0 .
$$

Simple algebraic calculations show that the $2 \times 2$-matrix

$$
\mathcal{M}(z)=\mathcal{A}_{\sharp \sharp}(z)-\mathcal{A}_{\sharp \bullet}(z) \mathcal{A}_{\bullet \bullet}(z)^{-1} \mathcal{A}_{\bullet \sharp}(z)
$$

inherits the symmetry and positiveness properties from the matrix $\mathcal{A}$ in (2.32), cf., [7], Sect. 2.4. By (2.33), it admits the representation

$$
\mathcal{M}(z)=z^{4} M z^{4}, \quad M=\mathcal{M}(1) .
$$

We denote eigenvalues of the matrix $M$ by

$$
0<m_{1} \leq m_{2},
$$

and the corresponding eigenvectors by $\mathcal{W}_{\sharp}^{1}$ and $\mathcal{W}_{\sharp}^{2}$ while their normalization will be clarified in Sect. 4.2.

\subsection{Power-Logarithmic Solutions}

Components of the eigenvectors $\mathcal{W}_{\sharp}^{j}$ are to be taken as coefficients of the power-law solutions $w_{1}$ and $w_{2}$ in the representation (2.10). We set

$$
w_{\sharp}^{j}(z)=z^{\kappa-5 / 2} \mathcal{W}_{\sharp}^{j} .
$$

Inserting (3.6) into (3.3) gives the following biquadratic equation for the exponent $\kappa$ :

$$
m_{j}\left(\kappa^{2}-\frac{49}{4}\right)\left(\kappa^{2}-\frac{25}{4}\right)=\Lambda|\varpi| .
$$

Hence,

$$
\kappa^{2}=\frac{49+25}{8} \pm \sqrt{\left(\frac{49-25}{8}\right)^{2}+\Lambda \frac{|\varpi|}{m_{j}}}=\frac{37}{4} \pm \sqrt{9+\Lambda \frac{|\varpi|}{m_{j}}} .
$$

Let us sort out the power-law solutions (3.6) generated by roots of Eq. (3.7). Thus, in the case

$$
\Lambda>\Lambda_{j}^{\dagger}:=m_{j} \frac{5^{2} 7^{2}}{4^{2}|\varpi|}=m_{j} \frac{1225}{16|\varpi|}
$$


Eq. (3.7) has two real and two pure imaginary roots

$$
\kappa_{j \pm}^{r e}= \pm \sqrt{\frac{37}{4}+\sqrt{9+\Lambda m_{j}^{-1}|\varpi|}}
$$

and

$$
\kappa_{j \pm}^{i m}= \pm i \sqrt{\sqrt{9+\Lambda m_{j}^{-1}|\varpi|}-\frac{37}{4}} .
$$

In the case

$$
\Lambda<\Lambda^{\dagger}=\min \left\{\Lambda_{1}^{\dagger}, \Lambda_{2}^{\dagger}\right\}=\Lambda_{1}^{\dagger},
$$

all roots of the biquadratic equations (3.7) with $j=1$ and $j=2$ are real and besides four of them are positive and four are negative. In the cases $\Lambda \in\left(\Lambda_{1}^{+}, \Lambda_{2}^{+}\right)$or $\Lambda \in\left(\Lambda_{2}^{+},+\infty\right)$ the equation has two or four purely imaginary roots. The case $\Lambda=\Lambda^{\dagger}$ will be considered below.

Using the roots (3.9) and (3.10), one readily finds out the power-law solutions (3.6) of the system (3.3). Moreover, the relation

$$
w_{\bullet}(z)=-\int \mathcal{A}_{\bullet \bullet}(z)^{-1} \mathcal{A}_{\bullet \sharp}(z) \partial_{z}^{2} w_{\sharp}(z) d z,
$$

which follows from (3.2), restores the column vectors of the solutions (2.11) under the conditions $\kappa \neq 3 / 2$ and $\kappa \neq 7 / 2$. If $\kappa=3 / 2$ or $\kappa=7 / 2$, the indefinite integral in (3.12) gives rise to logarithmical factors, i.e., $w_{\bullet}$ takes the form

$$
\left(\mathcal{W}_{3}^{1} \log z+\mathcal{W}_{3}^{0}, z^{-2} \mathcal{W}_{4}\right)^{\top} \quad \text { or } \quad\left(z^{2} \mathcal{W}_{3}, \mathcal{W}_{4}^{1} \log z+\mathcal{W}_{4}^{0}\right)
$$

Logarithms also appear in the case $\Lambda=\Lambda_{j}$, when null is a double root of Eq. (3.7). Then two corresponding solutions to the system (3.3) are

$$
w_{\sharp}^{j 0}(z)=z^{-5 / 2} \mathcal{W}_{\sharp}^{j} \quad \text { and } \quad w_{\sharp}^{j 1}(z)=z^{-5 / 2} \mathcal{W}_{\sharp}^{j} \log z .
$$

The column vectors $w_{\bullet}^{j 0}(z)$ and $w_{\bullet}^{j 1}(z)$ are found through the formula (3.12) wherein $w_{\bullet}^{j 1}(z)$ gains logarithm, too.

Finally, we write two evident constant solutions of the system (2.31)

$$
w^{30}=(0,0,1,0) \text { and } \quad w^{40}=(0,0,0,1),
$$

which admit the form (2.10), (2.11) with $\kappa_{3}=3 / 2$ and $\kappa_{4}=7 / 2$ but are not obtained by the reduction scheme in Sect. 3.1. Since this system is formally self-adjoint there exist two other solutions

$$
w^{p 1}(z)=z^{-\kappa_{p}-5 / 2}\left(\mathcal{W}^{p 1}, \mathcal{W}^{p 2}, z \mathcal{W}^{p 3}, z^{-1} \mathcal{W}^{p 4}\right) \quad \text { with } p=3,4
$$

No logarithm occurs in (3.16).

All power-logarithmic solutions to the system (2.31) have been constructed. 


\subsection{Elastic Waves in the Cusp}

We recall our splitting rule in Sect. 3.2, see (3.1), and observe that, under the restriction (3.8), the power-law solutions of the system (3.3) with complex exponents

$$
w_{\sharp}^{j \pm}(z)=z^{ \pm i \kappa-5 / 2} \mathcal{W}_{\sharp}^{j \pm}, \quad \kappa>0,
$$

generate the displacements fields $u^{j \pm}$ which are given by the formulas (2.2)-(2.4) and (2.21), where the role of the vector function (2.6) is played by

$$
w^{j \pm}(z)=z^{ \pm i \kappa-5 / 2}\left(\mathcal{W}_{1}^{j}, \mathcal{W}_{2}^{j}, z \mathcal{W}_{3}^{j \pm}, z^{-1} \mathcal{W}_{4}^{j \pm}\right)^{\top}
$$

and $w_{\bullet}^{j \pm}(z)$ has the form (3.12). Evaluating the deformation column vector (1.7) on the basis of formulas (2.2)-(2.4) and(2.21) provides the relation

$$
\varepsilon\left(u^{j \pm} ; y, z\right)=\left(Y(y)+D\left(\nabla_{y}, 0\right) X(y, z)\right) \mathcal{D}\left(\partial_{z}\right) w^{j \pm}(z)+\cdots
$$

Here and in what follows, ellipsis stands for inessential higher-order terms in asymptotic expansions. Thus, the linear elastic energy at the cross-section $\varpi(z)$ is equal to

$$
\begin{aligned}
E\left(u^{j \pm} ; z\right)= & \left.\frac{1}{2} \int_{\varpi(z)} \overline{\varepsilon\left(u^{j \pm} ; y, z\right)}\right)^{\top} \sigma\left(u^{j \pm} ; y, z\right) d y \\
= & \frac{1}{2}\left(\overline{\mathcal{D}\left(\partial_{z}\right) w^{j \pm}(z)}\right)^{\top} \int_{\varpi(z)}\left(D\left(\nabla_{y}, 0\right) X(y, z)+Y(y)\right)^{\top} A\left(D\left(\nabla_{y}, 0\right) X(y, z)\right. \\
& +Y(y)) d y \mathcal{D}\left(\partial_{z}\right) w^{j \pm}+\cdots \\
= & \frac{1}{2}\left(\overline{\mathcal{D}\left(\partial_{z}\right) w^{j \pm}(z)}\right)^{\top} \mathcal{A}(z) \mathcal{D}\left(\partial_{z}\right) w^{j \pm}+\cdots \\
= & \frac{1}{2}{\overline{\partial_{z}^{2} w_{\sharp}^{j \pm}(z)}}^{\top} \mathcal{M}(z) \partial_{z}^{2} w_{\sharp}^{j \pm}(z)+\cdots \\
= & \frac{1}{2 z}\left(\frac{25}{4}+\kappa^{2}\right)\left(\frac{49}{4}+\kappa^{2}\right)\left(\overline{\mathcal{W}_{\sharp}^{j \pm}}\right)^{\top} M \mathcal{W}_{\sharp}^{j \pm}+\cdots \\
= & \frac{1}{2 z}\left(\frac{25}{4}+\kappa^{2}\right)\left(\frac{49}{4}+\kappa^{2}\right) m_{j}\left|\mathcal{W}_{\sharp}^{j \pm}\right|^{2}+\cdots
\end{aligned}
$$

The fact that the linear energy is inversely proportional to the distance from the cusp tip $\mathcal{O}$, means that the bulk energy induced by the wave (3.17) in the cusp fragment

$$
\Pi_{K \rho}^{\rho}=\Pi_{K \rho} \backslash \bar{\Pi}_{\rho}=\left\{x \in \Pi_{d}: \rho<z<K \rho\right\}, \quad K=\mathrm{const}>1,
$$

does not depend on the distance $\rho$ from the tip $\mathcal{O}$, that is,

$$
\int_{\rho}^{K \rho} E(z) d z=\frac{1}{2 z}\left(\frac{25}{4}+\kappa^{2}\right)\left(\frac{49}{4}+\kappa^{2}\right) m_{j}\left|\mathcal{W}_{\sharp}^{j \pm}\right|^{2} \log K+\cdots .
$$

Thus, in the logarithmic scale, the total energy of the cusp fragment (3.21) gains negligible changes when $\rho$ tends to zero and the fragment $\Pi_{K \rho}^{\rho}$ approaches the tip $\mathcal{O}$. This observation characterizes $\Lambda$ in (3.8) as a point of the continuous spectrum of problem (1.10), (1.11) (compare with similar considerations in [24] in the case of a cylindrical waveguide). A mathematical argument appeals to singular Weyl sequences (see, e.g., [25, Sect. 9.2]) which had been constructed in $[1,7]$. 


\subsection{Propagation of Waves}

We accept the relationship (3.8) so that the system (2.31) admits a solution (3.18) with the complex exponent $\pm i \kappa-5 / 2$. Then, let us consider a harmonic in time wave $\operatorname{Re} \mathbf{u}^{j \pm}(t, x)$, where

$$
\mathbf{u}^{j \pm}(t, x)=e^{-i \omega t} u^{j \pm}(x), \quad \omega>0,
$$

and $u^{j \pm}$ is the displacement vector (2.2) generated by a solution (3.17) of the system (3.3), cf. Sect. 3.5. Due to (2.3), (2.4) and (2.10), (2.12) we have

$$
\left|u_{p}^{j \pm}(x)\right|=\mathcal{O}\left(z^{-5 / 2}\right), \quad p=1,2, \quad\left|u_{3}^{j \pm}(x)\right|=\mathcal{O}\left(z^{-3 / 2}\right) .
$$

In this way, the transverse oscillations dominate over the longitudinal one in the cusp, cf., Sect. 2.2. Furthermore, formulas (3.19) and (2.20), (2.24) show that the deformation column vector $\varepsilon\left(u^{j \pm}\right)$ as well as the stress column vector $\sigma\left(u^{j \pm}\right)=A \varepsilon\left(u^{j \pm}\right)$, see (1.8), get the order $\mathcal{O}\left(z^{-5 / 2}\right)$, and all four components of the solution (3.18) to the system (2.31) are involved into these column vectors.

The leading term in the asymptotic representation of the displacement field (3.23) converts into

$$
z^{5 / 2} \mathbf{u}_{\sharp}^{j \pm}(t, x)=z^{5 / 2}\left(u_{1}^{j \pm}(t, x), u_{2}^{j \pm}(t, x)\right) \sim \mathcal{W}_{\sharp}^{j \pm} e^{ \pm i \kappa\left(\log z \mp \kappa^{-1} \omega t\right)},
$$

cf., (3.1) and remains unchanged under the following relation between time and distance variables $t$ and $z$ :

$$
z=e^{ \pm \kappa^{-1} \omega t}
$$

This relation means that the wave $\mathbf{u}^{j-}$ propagates in direction to the tip $\mathcal{O}$, since in the case $t_{1}<t_{2}$ the corresponding coordinates $z_{1}$ and $z_{2}$ from (3.24) (with minus sign) meet the inequality $z_{2}<z_{1}$. Conversely, the wave $\mathbf{u}^{j+}$ propagates in direction from the tip of the cusp $\Pi_{d}$ to the solid $\Omega \backslash \Pi_{d}$. Moreover, in a certain (rather obscure) sense the waves $\mathbf{u}^{j-}$ and $\mathbf{u}^{j+}$ require infinite time to reach the tip and then to return to the massive part $\Omega \backslash \Pi_{d}$ of the body.

The above classification of waves (the outgoing wave $\mathbf{u}^{j-}$ and the incoming wave $\mathbf{u}^{j+}$ ) is mainly based on the classical Sommerfeld radiation principle, see, e.g., [26, 27]. However, it is known (see [28] as well as [29, Ch. 1], [30] and other publications), that in elastic waveguides, even of cylindrical shape, direction of the wave propagation according to Sommerfeld can differ from the direction of the energy transfer because of the opposite sign of the group velocity. In this way, the limiting absorption principle [26, 27] is usually applied although it does not work at the thresholds of the continuous spectrum (see a discussion in [29, Ch. 1] and an example in $[30, \S 5]$ ). Clearly, the Sommerfeld radiation principle also does not serve for our threshold case $\kappa=0$. Since we want to cover the whole continuous spectrum, in the next section we employ the universal approach which refers to the Umov-Poynting vector [31, 32] and the energy radiation conditions due to L. Mandelstam [33] (see also [29, Ch. 1] and [30]) and adapt them to wave propagation in cuspidal elastic solids. Furthermore, we will demonstrate in Sect. 5 that the corresponding radiation conditions guarantee a correct formulation of the problem in weighted spaces with detached asymptotics. 


\subsection{Notation and Terminology of Further Use}

In the one-dimensional model we will call oscillatory the complex-valued waves (3.18) with $\kappa>0$. In the case $\kappa=0$ these waves become standing while waves with the components (3.12) and (3.14) are resonant due to presence of the additional growing factor $\log z$; both the waves can be chosen real. Notice that we systematically ignore the factors $z^{-5 / 2}$ in $w_{\sharp}(z)$ and similar factors in $w_{\bullet}(z)$ whose role is to provide the linear energy (3.20) on the cross-section $\varpi(z)$ with the independence property discussed in Sect. 3.3. Without the weighting factors the introduced terminology is quite similar to the traditional terminology in cylindrical waveguides, cf. [26, 27, 29] and others.

This terminology also applies to the displacement fields $u$ defined according to (2.2)(2.4), (2.21) in the three-dimensional cusp (1.1). In this way, all above-mentioned waves make the elastic energy infinite in the intact set $\Pi_{d}$, see the representation (3.22) with $K \rightarrow+0$. The same property is attributed to waves initiated by the component (3.6) with $\kappa<0$ but in the case $\kappa>0$ the elastic energy computed in $\Pi_{d}$ for the corresponding threedimensional ansatz (2.2) becomes finite. The latter waves with $\kappa>0$ and $\kappa<0$ will be recognized as energy and non-energy waves.

To formulate the radiation conditions, we will need only three terms in the representation

$$
\begin{aligned}
\widehat{u}(y, z) & =U^{-2}(y, z)+U^{-1}(y, z)+U^{0}(y, z) \\
& =\sum_{j=1}^{2} w_{j}(z) e_{(j)}+\left(w_{3}(z)-\sum_{j=1}^{2} y_{j} \frac{\partial w_{j}}{\partial z}(z)\right) e_{(3)}+X(y, z) \mathcal{D}\left(\partial_{z}\right) w(z)
\end{aligned}
$$

of the three-dimensional displacement vector generated by a solution $w$ of the limit system (2.31) but only one term in the representation

$$
\widehat{\varepsilon}(\widehat{u} ; y, z)=\left(Y(y)+D\left(\nabla_{y}, 0\right) X(y, z)\right) \mathcal{D}\left(\partial_{z}\right) w(z)
$$

of the corresponding strain column vector while the stress column vector $\widehat{\sigma}(\widehat{u} ; y, z)$ is computed according to Hooke's law (1.8). The sum (3.25) is obtained by inserting (2.3), (2.4) and (2.21) into the truncated ansatz (2.2). We will calculate the strain tensor (3.26) in Sect. 4.3, see (4.9).

In the paper [7] a procedure is described to construct the complete asymptotic series for these elastic fields. Moreover, according to Sect. 4.9 in [7], any solution $w$ of the system (2.31) gives rise to the three-dimensional displacement vector $u$ which has the sum (3.25) as the main asymptotic term and satisfies the differential equations (1.2) in $\Pi_{d^{\prime}}$ and the boundary conditions (1.3) on $\Gamma_{d^{\prime}}$ with some $d^{\prime} \in(0, d]$ but of course no boundary condition is imposed on $u$ at the cross-section $\overline{\omega\left(d^{\prime}\right)}=\partial \Pi_{d^{\prime}} \backslash \Gamma_{d^{\prime}}$.

More exactly, a formal asymptotic solution to the problem (1.10), (1.11)

$$
u_{k}(y, z) \approx \sum_{j \geq 0}\left(u_{k j}^{-2}(\eta, z)+u_{k j}^{-1}(\eta, z)+u_{k j}^{0}(\eta, z)+u_{k j}^{1}(\eta, z)\right)
$$

is constructed in [7], where coefficients of the series actually are written under the coordinate change

$$
y \mapsto \eta=\frac{y}{z^{2}}, \quad z \mapsto t=\frac{1}{z} .
$$


Here, $k=1, \ldots, 12$, the functions $u_{k 0}^{p}(\eta, z), p=-2,-1,0,1$, coincide with the functions (2.7), (2.8), where the function $w$ is chosen according to (3.6) and for the exponent $\kappa$ one of 12 possibilities described in Sect. 3.2 is taken into account. Other terms with indexes $j>0$ get additional factors $z^{j}$ and possibly $(\log z)^{p_{j}}$. Let

$$
u_{k}^{N}(y, z)=\sum_{j \leq N}\left(u_{k j}^{-2}(\eta, z)+u_{k j}^{-1}(\eta, z)+u_{k j}^{0}(\eta, z)+u_{k j}^{1}(\eta, z)\right) .
$$

In [7, Sect. 4.9] it was proved that for each $N$ there exists a solution of the problem (1.10), (1.11) in $\Pi_{d^{\prime}}$ with a certain small $d^{\prime}>0$ in the form

$$
\widehat{u}^{k}(y, z)=u_{k}^{N}(y, z)+\widetilde{u}_{k}^{N}(y, z)
$$

where $\widetilde{u}_{k}^{N}$ admits the estimate

$$
\int_{\Pi_{d}}\left(\left|\tilde{u}_{k}^{N}(y, z)\right|^{2}+\left|\nabla_{z} \tilde{u}_{k}^{N}(y, z)\right|^{2}\right) z^{\gamma-N} d y d z<\infty,
$$

with an exponent $\gamma$ independent of $N$.

Theorem 1 There exists a positive constant $\delta$ with the following property. If a solution $u$ to the problem (1.10), (1.11) restricted on the cusp $\Pi_{d}$ satisfies the condition

$$
\int_{\Pi_{d}}\left(|u(y, z)|^{2}+\left|\nabla_{x} u(y, z)\right|^{2}\right) e^{-\delta / z} d y d z<\infty,
$$

then this solution admits the asymptotic representation in the form

$$
u(y, z)=\sum_{k=1}^{12} c_{k} \widehat{u}^{k}(y, z)+\widetilde{\widetilde{u}}(y, z),
$$

where $\widehat{u}^{k}$ are the vector functions (3.29), (3.30), the remainder enjoys the decay property

$$
\int_{\Pi_{d}}\left(|\widetilde{\tilde{u}}(y, z)|^{2}+\left|\nabla_{x} \widetilde{\widetilde{u}}(y, z)\right|^{2}\right) e^{\delta / z} d y d z<\infty,
$$

and the coefficients in (3.32) meet the estimate

$$
\sum_{k=1}^{12}\left|c_{k}\right| \leq C\left(\int_{\Omega \backslash \Pi_{d}}|u(y, z)|^{2} d y d z\right)^{1 / 2} .
$$

Proof Using variables $\eta$ and $t=z^{-1}$, we can write the problem (1.10), (1.11) restricted on the cusp $\Pi_{d}$ as a variational problem with constant coefficients in a half-cylinder perturbed by an differential operator with coefficients vanishing at infinity, see Sect. 10.8 [8] for an abstract treatment of such variational problems. Now we want to apply Theorem 9.3.2 together with Remark 10.8.14 in [8]. In our case the spectrum of the operator pencil corresponding to the Neumann problem for the elasticity operator in the cylinder $\varpi \times \mathbb{R}$ consists of eigenvalues of finite algebraic multiplicity and there is only one eigenvalue $\lambda=0$ of multiplicity 12 on the imaginary axis (we note that the notation in [8] uses the additional factor $i$ in the definition of eigenvalues). Moreover, there exist $\delta>0$ such that there is no eigenvalues 
in the strip $\{\lambda \in \mathbb{C}:|\operatorname{Im} \lambda| \leq \delta\}$ with exception of $\lambda=0$. Using the notation from Theorem 9.3.2 [8], we can set $k_{+}^{(1)}=k_{-}^{(2)}=0, k_{-}^{(1)}=\delta, k_{+}^{(2)}=-\delta$. Moreover, we observe that the space $\mathcal{X}(L)$ coincides with the linear combination of the solutions $\widehat{u}^{k}$ constructed above. ${ }^{3}$ Now the reference to Theorem 9.3.2 [8] proves our theorem.

It is worth to observe that the expression (3.25) with the constant solutions (3.15) converts into the rigid motions

$$
\widehat{u}^{30}(y, z)=(0,0,1)^{\top} \text { and } \widehat{u}^{40}(y, z)=\theta(y),
$$

a longitudinal displacement and a rotation about the $z$-axis, respectively, but, for any $\Lambda>0$, the whole vector functions $u^{30}$ and $u^{40}$ mentioned in the previous paragraph differ from (3.34) because rigid motion does not satisfy the problem (1.10), (1.11). Inside the continuous spectrum the transverse displacements $(1,0,0)^{\top}$ and $(0,1,0)^{\top}$, being rigid motions too, turn into oscillatory waves which will be examined in the next section.

Finally, we mention that a power-law solution with the components (2.10), (2.11) generates a three-dimensional displacement field $u$ with the finite energy functional

$$
E\left(u ; \Pi_{d}\right)=\int_{0}^{d} E(z) d z
$$

if and only if

$$
\operatorname{Re} \kappa>0 \text {. }
$$

It is remarkable that, for the oscillating waves (3.19), the differences $u^{j \pm}-\widehat{u}^{j \pm}$ again keeps the energy functional $E\left(u ; \Pi_{d^{\prime}}\right)$ finite.

\section{Energy Radiation Conditions}

\subsection{The Umov-Poynting Vector}

The vector $J(u ; t, x)$ of energy transfer was introduced by N.A. Umov in [31] for elasticity and acoustic problems and by J.H. Poynting in [32] for electro-magnetic problems. It describes the energy flux through a part $\Sigma=\partial \Theta \backslash \partial \Omega$ of the boundary of a chosen subdomain $\Theta$ in $\Omega$, in other words,

$$
-\frac{d}{d t} E(\mathbf{u} ; t, \Theta)=\int_{\Sigma} n(x)^{\top} J(\mathbf{u} ; t, x) d s_{x},
$$

where $n(x)$ stands for the unit outward normal, $E(\mathbf{u} ; t, \Theta)$ is the total (elastic plus kinematic) energy,

$$
E(\mathbf{u} ; t, \Theta)=\frac{1}{2}(A D(\nabla) \operatorname{Re} \mathbf{u}, D(\nabla) \operatorname{Re} \mathbf{u})_{\Theta}+\frac{1}{2} \rho\left(\frac{\partial}{\partial t} \operatorname{Re} \mathbf{u}, \frac{\partial}{\partial t} \operatorname{Re} \mathbf{u}\right)_{\Theta},
$$

\footnotetext{
${ }^{3}$ This space is defined as the space of solutions to (1.10), (1.11) on $\Pi_{d}$ subject to (3.31), which are factorized by solutions to (1.10), (1.11) on $\Pi_{d}$ satisfying (3.33). The dimension of this space is 12 as it is shown in [8].
} 
compare with the quadratic form (1.13). According to formula (3.23) for $\mathbf{u}$, we have

$$
\begin{aligned}
\frac{d}{d t} E(\mathbf{u} ; t, \Theta)= & \omega(A D(\nabla) \operatorname{Re} \mathbf{u}, D(\nabla) \operatorname{Im} \mathbf{u})_{\Theta}-\rho \omega^{3}(\operatorname{Re} \mathbf{u}, \operatorname{Im} \mathbf{u})_{\Theta} \\
= & \omega \int_{\Theta} \operatorname{Im} \mathbf{u}(t, x)^{\top}\left(D(-\nabla)^{\top} A D(\nabla) \operatorname{Re} \mathbf{u}(t, x)-\rho \omega^{2} \operatorname{Re} \mathbf{u}(t, x)\right) d x \\
& +\omega \int_{\Sigma} \operatorname{Im} \mathbf{u}(t, x)^{\top} D(n(x))^{\top} A D(\nabla) \operatorname{Re} \mathbf{u}(t, x) d s_{x}
\end{aligned}
$$

The last integral over $\Theta$ is null due to the differential equations (1.10) and the surface integral can be reduced to the surface $\Sigma$ because the integrand vanishes on $\partial \Theta \backslash \Sigma \subset \partial \Omega$ due to the boundary condition (1.11) (clearly, there is no radiation of energy through traction-free surfaces). Comparing (4.1) and (4.2), we see that components of the Umov-Poynting vector are given by

$$
J_{j}(\mathbf{u} ; t, x)=-\omega \operatorname{Im} \mathbf{u}(t, x)^{\top} D\left(e_{(j)}\right)^{\top} A D(\nabla) \operatorname{Re} \mathbf{u}(t, x), \quad j=1,2,3 .
$$

\subsection{Energy Radiation Conditions}

The energy radiation conditions due to L. Mandelstam [33] (see also the publications $[29,30]$ and many others) associate direction of wave propagation with direction of the energy transfer. To be more precise, the wave $\mathbf{u}(t, x)=e^{-i \omega t} u(x)$, cf. (3.23), propagates in the direction to the cusp tip $\mathcal{O}$ provided the $z$-axis projection $\mathbf{J}_{3}(\mathbf{u} ; z)$ of the mean value in $t \in(0,2 \pi / \omega)$ of the Umov-Poynting vector

$$
\begin{aligned}
\mathbf{J}_{3}(\mathbf{u} ; z)= & i \frac{\omega}{2 \pi} \omega \int_{0}^{2 \pi / \omega} \int_{\varpi(z)} \frac{1}{2}\left(e^{-i \omega t} u(y, z)-e^{i \omega t} \overline{u(y, z)}\right) \\
& \times D\left(e_{(3)}\right)^{\top} A D(\nabla) \frac{1}{2}\left(e^{-i \omega t} u(y, z)+e^{i \omega t} \overline{u(y, z)}\right) d y d t \\
= & i \frac{\omega}{4} \int_{\varpi(z)}\left(u(y, z)^{\top} D\left(e_{(3)}\right)^{\top} A D(\nabla) \overline{u(y, z)}\right. \\
& \left.-\overline{u(y, z)}^{\top} D\left(e_{(3)}\right)^{\top} A D(\nabla) u(y, z)\right) d y=-i \frac{\omega}{4} q_{z}(u, u)
\end{aligned}
$$

is negative. Here, we introduced the symplectic, that is, sesquilinear and anti-Hermitian, form

$$
\begin{aligned}
q_{z}(u, v)= & \int_{\varpi(z)}\left(\overline{v(y, z)}^{\top} D\left(e_{(3)}\right)^{\top} A D(\nabla) u(y, z)\right. \\
& \left.-u(u, z)^{\top} D\left(e_{(3)}\right)^{\top} A D(\nabla) \overline{v(y, z)}\right) d y,
\end{aligned}
$$

which appears as a surface integral in Green's formula on $\Pi_{d}^{\rho}$. The inequality $\operatorname{Im} q_{z}(u, u)<0$ means that the wave $u$ transports the total energy in the direction of the cusp tip $\mathcal{O}$ while the inequality $\operatorname{Im} q_{z}(u, u)>0$ indicates the opposite direction of energy transfer.

\subsection{Normalization of Oscillatory Waves}

Let us calculate the limit

$$
q_{0}\left(u^{j \pm}, u^{j \pm}\right)=\lim _{z \rightarrow+0} q_{z}\left(u^{j \pm}, u^{j \pm}\right)
$$


for the field $u^{j \pm}$ generated by the solution (3.18) of the system (2.31). In the representation formula (3.25) for the displacement field it is sufficient to take into account two terms (2.3) and (2.4) only, that is,

$$
u^{j \pm}(x)=U^{-2}(z)+U^{-1}(y, z)+\mathcal{O}\left(z^{-1 / 2}\right),
$$

but in comparison with (3.26) the representation formula for the deformation column vector must also involve the additional term (2.21), namely

$$
\begin{aligned}
\varepsilon\left(u^{j \pm}\right)= & D\left(\nabla_{y}, 0\right) U^{-2}+\left(D\left(\nabla_{y}, 0\right) U^{-1}+D\left(0, \partial_{z}\right) U^{-2}\right) \\
& +\left(D\left(\nabla_{y}, 0\right) U^{0}+D\left(0, \partial_{z}\right) U^{-1}\right)+\left(D\left(\nabla_{y}, 0\right) U^{1}+D\left(0, \partial_{z}\right) U^{0}\right)+\cdots .
\end{aligned}
$$

The first two terms in (4.8) vanish by the definitions (2.3) and (2.4) and the third one can be converted by means of (2.21) and (2.18), (2.19). These lead to

$$
\begin{aligned}
\varepsilon\left(u^{j \pm} ; x\right)= & \left(Y(y)+D\left(\nabla_{y}, 0\right) X(y, z)\right) \mathcal{D}\left(\partial_{z}\right) w^{j \pm}(z) \\
& +\left(D\left(\nabla_{y}, 0\right) U^{1}(y, z)+D\left(0, \partial_{z}\right) U^{0}(y, z)\right)+\mathcal{O}\left(z^{-1 / 2}\right) .
\end{aligned}
$$

Since the area of the cross-section $\varpi(z)$ is $O\left(z^{4}\right)$, the achieved accuracy in (4.7) and (4.9) is sufficient to calculate the limit (4.6).

We note that according to (1.9) and (2.3), see also (2.16),

$$
D\left(e_{(3)}\right) U^{-2}(z)=\sum_{p=1}^{2} w_{p}^{j \pm}(z) D\left(\nabla_{y}, 0\right) y_{p} e_{(3)}
$$

and, hence,

$$
\begin{aligned}
& \int_{\varpi(z)}\left(\overline{U^{-2}}\right)^{\top} D\left(e_{(3)}\right)^{\top} A\left(Y+D\left(\nabla_{y}, 0\right) X\right) d y \\
& =\sum_{p=1}^{2} \overline{w_{p}^{j \pm}} \int_{\varpi(z)}\left(D\left(\nabla_{y}, 0\right) y_{p} e_{(3)}\right)^{\top} A\left(Y+D\left(\nabla_{y}, 0\right) X\right) d y=0 .
\end{aligned}
$$

The last equality results from integrating by parts and taking (2.22) into account. Moreover,

$$
\begin{aligned}
& D\left(e_{(3)}\right) U^{-1}(y, z)=Y(y) \mathcal{D}^{\prime}\left(\partial_{z}\right) w^{j \pm}, \\
& \mathcal{D}^{\prime}\left(\partial_{z}\right)=\operatorname{diag}\left\{\partial_{z}, \partial_{z}, 1,1\right\}, \quad \mathcal{D}\left(\partial_{z}\right)=\mathcal{D}^{\prime}\left(\partial_{z}\right) \partial_{z} .
\end{aligned}
$$

Therefore, in view of the definition (2.32), we have

$$
\begin{aligned}
& \int_{\varpi(z)}{\overline{U^{1}(y, z)}}^{\top} D\left(e_{(3)}\right)^{\top} A\left(D\left(\nabla_{y}, 0\right) X(y, z)+Y(y)\right) d y \mathcal{D}\left(\partial_{z}\right) w^{j \pm}(z) \\
& \quad=\left(\overline{\mathcal{D}^{\prime}\left(\partial_{z}\right) w^{j \pm}(z)}\right)^{\top} \int_{\varpi(z)} Y(y)^{\top} A\left(D\left(\nabla_{y}, 0\right) X(y, z)+Y(y)\right) d y \mathcal{D}\left(\partial_{z}\right) w^{j \pm}(z) \\
& =\left(\overline{\mathcal{D}^{\prime}\left(\partial_{z}\right) w^{j \pm}(z)}\right)^{\top} \mathcal{A}(z) \mathcal{D}\left(\partial_{z}\right) w^{j \pm}(z)=\overline{\left(\partial_{z} w_{\sharp}^{j \pm}(z)\right)} \mathcal{M}(z) \partial_{z}^{2} w_{\sharp}^{j \pm}(z) .
\end{aligned}
$$


Finally,

$$
\begin{aligned}
& \int_{\varpi(z)}\left(\overline{U^{2}}\right)^{\top} D\left(e_{(3)}\right)^{\top} A\left(D\left(\nabla_{y}, 0\right) U^{1}+D\left(0, \partial_{z}\right) U^{0}\right) d y \\
& =\sum_{p=1}^{2} \overline{w_{p}^{j \pm}} \int_{\varpi(z)}\left(D\left(e_{(3)}\right) e_{(p)}\right)^{\top} A\left(D\left(\nabla_{y}, 0\right) U^{1}+D\left(0, \partial_{z}\right) U^{0}\right) d y \\
& =\sum_{p=1}^{2} \overline{w_{p}^{j \pm}} \int_{\varpi(z)}\left(D\left(\nabla_{y}, 0\right) y_{p} e_{(3)}\right)^{\top} A\left(D\left(\nabla_{y}, 0\right) U^{1}+D\left(0, \partial_{z}\right) U^{0}\right) d y \\
& =\sum_{p=1}^{2} \overline{w_{p}^{j \pm}} e_{(3)}^{\top}\left(-\int_{\varpi(z)} y_{p} D\left(\nabla_{y}, 0\right)^{\top} A\left(D\left(\nabla_{y}, 0\right) U^{1}+D\left(0, \partial_{z}\right) U^{0}\right) d y\right. \\
& \left.+\int_{\partial \varpi(z)} y_{p} D(v, 0)^{\top} A\left(D\left(\nabla_{y}, 0\right) U^{1}+D\left(0, \partial_{z}\right) U^{0}\right) d s_{y}\right) \\
& =\sum_{p=1}^{2} \overline{w_{p}^{j \pm}} e_{(3)}^{\top}\left(\int_{\varpi(z)} y_{p} D\left(0, \partial_{z}\right)^{\top} A\left(D\left(\nabla_{y}, 0\right) U^{0}+D\left(0, \partial_{z}\right) U^{-1}\right) d y\right. \\
& \left.+2 z \int_{\partial \varpi(z)} y_{p} D\left(0, z^{-2} y^{\top} v\right)^{\top} A\left(D\left(\nabla_{y}, 0\right) U^{0}+D\left(0, \partial_{z}\right) U^{-1}\right) d s_{y}\right) \\
& =\sum_{p=1}^{2} \overline{w_{p}^{j \pm}} \partial_{z} \int_{\varpi(z)}\left(D\left(e_{(3)}\right) e_{(3)} y_{p}\right)^{\top} A\left(D\left(\nabla_{y}, 0\right) U^{0}+D\left(0, \partial_{z}\right) U^{-1}\right) d y \mathcal{D}\left(\partial_{z}\right) w^{j \pm} \text {. }
\end{aligned}
$$

These calculations are performed similarly to (2.28) and (2.29) by recalling the relation (2.16) together with the problem (2.15) at $q=1$ and integrating by parts. As a result, the factor in front of $A$ in the last integral converts into rows of the matrix $Y(y)^{\top}$ from (2.20) with minus sign. In this way, the expression (4.10) coincides with

$$
-{\overline{w_{\sharp}^{j \pm}(z)}}^{\top} \partial_{z}\left(\mathcal{A}_{\sharp \sharp}(z) \partial_{z}^{2} w_{\sharp}^{j \pm}(z)+\mathcal{A}_{\sharp \bullet}(z) \partial_{z} w_{\bullet}^{j \pm}(z)\right)=-{\overline{w_{\sharp}^{j \pm}(z)}}^{\top} \partial_{z} \mathcal{M}(z) \partial_{z}^{2} w_{\sharp}^{j \pm}(z) .
$$

We again use the notion introduced in (3.1). We further have

$$
\begin{aligned}
q_{z}\left(u^{j \pm}, u^{j \pm}\right) & =Q_{z}\left(w_{\sharp}^{j \pm}, w_{\sharp}^{j \pm}\right)-\overline{Q_{z}\left(w_{\sharp}^{j \pm}, w_{\sharp}^{j \pm}\right)}, \\
Q_{z}\left(w_{\sharp}^{j \pm}, w_{\sharp}^{j \pm}\right) & =-{\overline{w_{\sharp}^{j \pm}(z)}}^{\top} \partial_{z} \mathcal{M}(z) \partial_{z}^{2} w_{\sharp}^{j \pm}(z)+{\overline{\partial_{z} w_{\sharp}^{j \pm}(z)}}^{\top} \mathcal{M}(z) \partial_{z}^{2} w_{\sharp}^{j \pm}(z)
\end{aligned}
$$

and, owing to (3.17), we have

$$
q_{0}\left(u^{j \pm}, u^{j \pm}\right)= \pm 72 i \kappa m_{j}\left|\mathcal{W}_{\sharp}^{j}\right|^{2} .
$$

Now the normalization

$$
\left|\mathcal{W}_{\sharp}^{j}\right|=\frac{1}{6}\left(2 \kappa m_{j}\right)^{-1 / 2}
$$


of the eigenvectors of the $2 \times 2$-matrix $M$ from (3.5) provides the orthogonality and normalization conditions

$$
q_{0}\left(u^{j \pm}, u^{j \pm}\right)= \pm i, \quad q_{0}\left(u^{j \pm}, u^{j \mp}\right)=0,
$$

where the second equality follows from the obvious relation

$$
w_{\sharp}^{j \pm}(z)=\overline{w_{\sharp}^{j \mp}(z)} .
$$

\subsection{Logarithmic Packets of Standing and Resonance Waves}

Comparing the relations (4.3) and (4.11), we see that

$$
\mathbf{J}_{3}\left(e^{-i \omega t} u^{j \pm} ; z\right)= \pm \frac{\omega}{4}+o(1) \quad \text { as } z \rightarrow+0,
$$

i.e., according to the Mandelstam energy principle, the wave $u^{j-}$ propagates to the tip of the cusp, and $u^{j+}$ propagates from the tip. This classification does not differ from that observed in Sect. 3.4 due to the Sommerfeld principle. As was mentioned, the latter principle is not applicable for the standing wave $u^{j 0}$ as well as the resonance wave $u^{j 1}$, constructed according to (2.2) with the help of the power-law solution $w^{j 0}$ and the power-logarithmic solution $w^{j 1}$ to the system (3.3) at the threshold $\Lambda=\Lambda_{j}$, see (3.8) and (3.6), (3.13), (3.14) respectively. Furthermore, since solutions $w^{j p}$ are real-valued, we have the equalities

$$
q_{0}\left(u^{j 0}, u^{j 0}\right)=0, \quad q_{0}\left(u^{j 1}, u^{j 1}\right)=0,
$$

which means that the waves $u^{j 0}$ and $u^{j 1}$ do not transport energy at all. At the same time, repeating the above calculations yields

$$
q_{0}\left(u^{j 0}, u^{j 1}\right)=\frac{23}{2} m_{j}\left|\mathcal{W}_{\sharp}^{j}\right|^{2} .
$$

Hence, the normalization of the eigenvectors of the matrix $M$

$$
\left|\mathcal{W}_{\sharp}^{j}\right|=\left(\frac{2 m_{j}}{23}\right)^{1 / 2}
$$

gives us the formulas

$$
q_{0}\left(u^{j 0}, u^{j 1}\right)=-q\left(u^{j 1}, u^{j 0}\right)=1 .
$$

Following [34] and [14, Ch. 6], we introduce the wave packets

$$
w_{\sharp}^{j \pm}(z)=\frac{1}{\sqrt{2}}(1 \mp i \log z) z^{-5 / 2} \mathcal{W}_{\sharp}^{j}
$$

and note that according to (4.13) and (4.14), the orthogonality and normalization conditions (4.11) are valid as well. We see that the Mandelstam principle services even for the threshold situation, detecting the packet $u^{j-}$, generated by the solution $w_{\sharp}^{j-}$ of the system (3.3) as propagating to the cusp tip, and the packet $u^{j+}$ in the opposite direction. In other words, the corresponding three-dimensional waves $u^{j-}$ and $u^{j+}$ must be called outgoing and incoming, respectively. 


\subsection{Packets of Non-energy Waves}

Dealing with the constant energy waves (3.15) and didymous non-energy waves (3.16) which are real-valued, we immediately arrive at the formulas (4.13) with $j=3$, 4. At the same time, by a proper choice of the constant vectors $\mathcal{W}^{p}=\left(\mathcal{W}^{p 1}, \ldots, \mathcal{W}^{p 4}\right)^{\top}$ in (3.16), determined up to a multiplicative constant, we achieve the relations (4.14) with $j=3,4$. The latter has a clear mechanical reason because the three-dimensional realizations $u^{31}$ and $u^{41}$ of the power-law solutions (3.16) exhibit nothing but a longitudinal force and a torque about the $z$-axis. Finally, the analogous to (4.15) formulas with $j=3,4$

$$
w^{j \pm}(z)=\frac{1}{\sqrt{2}}\left(w^{j 0}(z) \mp i w^{j 1}(z)\right)
$$

define non-energy wave packets which are recognized by the Mandelstam radiation principle as outgoing (minus) and incoming (plus).

The same structure (4.16) applies to the energy $(\kappa>0)$ and non-energy $(\kappa<0)$ waves (3.6), (3.12) generated by real roots of the quadratic equation (3.7) in the case of its positive right-hand side. Namely, fixing a power-law solution $w^{j 0}=\left(w_{\sharp}^{j 0}, w_{\bullet}^{j 0}\right)$ with some root $\kappa_{j}>0$ we find its "companion" $w^{j 1}=\left(w_{\sharp}^{j 1}, w_{\bullet}^{j 1}\right)$ with the root $-\kappa_{j}<0$ such that the relations (4.13) and (4.14) are valid. Now the definition (4.16) gives us the outgoing $w^{j-}$ and incoming $w^{j+}$ waves in the one-dimensional model which in turn produce the three-dimensional waves $u^{j-}$ and $u^{j+}$ in the cusp (1.1).

It should be mentioned that the packets (4.16) are artificial objects needed for intermediate calculations in the next section.

\section{Solvability of the Elasticity Problem in Domains with Cusps}

\subsection{The Spectral Parameter is Below the Cutoff Value}

The interval $\left(0, \Lambda^{\dagger}\right)$, cf., (3.11), below the continuous spectrum (1.1) contains only points of the discrete spectrum $\wp_{d i}$ with finite total multiplicity \# $\wp_{d i}$. Thus, the solvability theorem on the elasticity problem (1.17), (1.18) in the variational form

$$
a(u, v ; \Omega)-\Lambda(u, v)_{\Omega}=F(v) \quad \forall v \in \mathcal{W}(\Omega)
$$

reads in the standard way, although in the integral identity (5.1) the energy space $\mathcal{W}(\Omega)$ with the norm (1.15) differs from the traditional Sobolev space $H^{1}(\Omega)^{3}$. By $\mathcal{L}_{\Lambda}$, we denote the space of eigenmodes, that is, solutions $w \in \mathcal{W}(\Omega)$ of the homogeneous $(F=0)$ variational problem (5.1) with the spectral parameter $\Lambda$ or the boundary value problem (1.10), (1.11).

Proposition 1 Let $F \in \mathcal{W}(\Omega)^{*}$ be an anti-linear continuous functional in the space $\mathcal{W}(\Omega)$ such that

$$
F(w)=0 \quad \forall w \in \mathcal{L}_{\Lambda} .
$$

Then the problem (5.1) with the parameter $\Lambda \in\left(0, \Lambda^{\dagger}\right)$ has a solution $u \in \mathcal{W}(\Omega)$ which is determined up to an addendum in $\mathcal{L}_{\Lambda}$, an eigenmode. The orthogonality conditions

$$
(u, w)_{\Omega}=0 \quad \forall w \in \mathcal{L}_{\Lambda}
$$


make the solution unique and assure the estimate

$$
\|u ; \mathcal{W}(\Omega)\| \leq c\left\|F ; \mathcal{W}(\Omega)^{*}\right\|
$$

with a factor $c$ which depends on the domain $\Omega$, the elastic moduli matrix $A$, and the spectral parameter $\Lambda$ but is independent of the functional $F$.

In view of the weighted anisotropic Korn inequality (1.16), see [1, 10], which predicts a possible behavior of the energy solutions $u \in \mathcal{W}(\Omega)$ at the tip $\mathcal{O}$ of the cusp $\Pi_{d}$, the particular functional on the right-hand side of (5.1)

$$
F(v)=(f, v)_{\Omega}+(g, v)_{\partial \Omega}
$$

with the volume force $f$ and the surface loading $g$ in the elasticity problem (1.17), (1.18) becomes continuous in $\mathcal{W}(\Omega)$ provided

$$
f_{k} \in L^{2}(\Omega), \quad r^{-1} g_{k} \in L^{2}(\partial \Omega), \quad k=1,2, \quad r f_{3} \in L^{2}(\Omega), \quad g_{3} \in L^{2}(\partial \Omega) .
$$

Notice that, owing to shrinking of the cusp cross-section as $z \rightarrow+0$, the inclusions (5.5) allows for growing right-hand sides in the problem (1.17), (1.18), namely the following relations can be valid with any exponent $\tau<5 / 2$ :

$$
\begin{aligned}
& f_{k}(x)=O\left(z^{-\tau}\right), \quad g_{k}(x)=O\left(z^{2-\tau}\right), \quad k=1,2, \\
& f_{3}(x)=O\left(z^{-1-\tau}\right), \quad g_{3}(x)=O\left(z^{1-\tau}\right) \quad \text { as } z \rightarrow+0 .
\end{aligned}
$$

Finally, we describe the behavior of elastic fields satisfying the problem (1.17), (1.18) with external loadings applied at a distance from the cusp tip $\mathcal{O}$, for example,

$$
\operatorname{supp} f \subset \bar{\Omega} \backslash \overline{\Pi_{d}}, \quad \operatorname{supp} g \subset \Gamma \backslash \overline{\Pi_{d}} .
$$

The restrictions (5.6), of course, are met in this case. The following relations have been verified in [7] but also can be formally derived from the asymptotic ansätze (2.2)-(2.4), (2.21) and (3.19) with the main asymptotic terms (2.10), (2.11):

$$
\begin{aligned}
u_{k}(x) & =O\left(z^{\kappa_{\min }(\Lambda)-5 / 2}\right), \quad k=1,2, \quad u_{3}(x)=O\left(z^{\kappa_{\min }(\Lambda)-3 / 2}\right), \\
\varepsilon(u ; x) & =O\left(z^{\kappa_{\min }(\Lambda)-5 / 2}\right) \quad \text { as } z \rightarrow+0,
\end{aligned}
$$

where

$$
\kappa_{\min }(\Lambda)=\min \left\{\kappa_{\min }^{r e}(\Lambda), 3 / 2,7 / 2\right\}
$$

and $\kappa_{\min }^{r e}(\Lambda)$ is the minimum among all positive roots of the biquadratic equations (3.7), $j=1,2$ (see Sect. 3.2 and recall that, under the restriction $\Lambda<\Lambda^{\dagger}$, each of those equations has two positive and two negative roots). Notice that $\kappa_{3}=3 / 2$ and $\kappa_{4}=7 / 2$ are the exponents in the constant power-law solutions (3.15) while $7 / 2$, of course, can be omitted in (5.8). 


\subsection{Embedded Eigenvalues and Trapped Modes}

The spectral parameter $\Lambda \geq \Lambda^{\dagger}$ belonging to the continuous spectrum $\wp_{c o}$ also can be an eigenvalue of the problem (1.10), (1.11). In other words, it can fall into the point spectrum $\wp_{p o}$. Examples of non-empty set $\wp_{p o}$ are given in [1]. Let us present a simplified version concerning the isotropic solid $\Omega$, see Sect. 2.6, with the rotational symmetry around the $z$-axis, cf., Fig. 1 . In particular, $\varpi$ is the unit disk $\mathbb{D}=\left\{y \in \mathbb{R}^{2}:|y|<1\right\}$. Let $(r, \varphi) \in \mathbb{R}_{+} \times(0,2 \pi)$ be the polar coordinate system in the $y$-plane and let $\Omega_{\varangle}$ be a sector in $\Omega$ of the opening $\pi / 3$,

$$
\Omega_{\varangle}=\{x \in \Omega: \varphi \in(0, \pi / 3)\}
$$

with the truncation surfaces $\Sigma_{\psi}=\{x \in \Omega: \varphi=\psi\}$, where $\psi=0$ and $\psi=\pi / 3$. We denote by $(1.10)_{\varangle}$ and $(1.11)_{\varangle}$ the differential equations (1.10) and the boundary conditions (1.11) restricted on the set (5.9) and its lateral surface $\Gamma_{\varangle}=\{x \in \Gamma: \varphi \in(0, \pi / 3)\}$, respectively. The corresponding problem is closed by the following artificial boundary conditions:

$$
\begin{gathered}
u_{n}=0, \quad \sigma_{s^{j} n}=0, \quad j=1,2, \quad \text { on } \Sigma_{0}, \\
\sigma_{n n}=0, \quad u_{s^{j}}=0, \quad j=1,2, \quad \text { on } \Sigma_{\pi / 3} .
\end{gathered}
$$

Here, $n$ and $s^{1}, s^{2}$ are the unit normal and perpendicular tangent vectors on the plane surface $\Sigma_{\psi}$ while projections of the displacement vector $u$ and the stress tensor $\sigma(u)$ on these axes are listed in (5.10) and (5.11).

The variational formulation of the spectral problem $(1.10)_{\varangle},(1.11)_{\varangle},(5.10),(5.11)$ refers to the integral identity

$$
a\left(u, v ; \Omega_{\varangle}\right)=\Lambda_{\varangle}(u, v)_{\Omega_{\varangle}} \quad \forall v \in \mathcal{W}_{\varangle}\left(\Omega_{\varangle}\right)
$$

and involves the following subspace of the energy space with the norm (1.15):

$$
\mathcal{W}_{\varangle}\left(\Omega_{\varangle}\right)=\left\{u \in \mathcal{W}\left(\Omega_{\varangle}\right): u_{n}=0 \text { on } \Sigma_{0}, u_{s}=0 \text { on } \Sigma_{\pi / 3}, j=1,2\right\} .
$$

The first distinguishing feature of the artificial boundary conditions (5.10), (5.11) proposed in [35] is the weighted Korn inequality, a simplified version of which is verified in the next assertion.

\section{Proposition 2 The anisotropic weighted inequality}

$$
\left\|r^{-2} u ; L^{2}\left(\Omega_{\varangle}\right)\right\|^{2} \leq K_{\varangle}\left\|D(\nabla) u ; L^{2}\left(\Omega_{\varangle}\right)\right\|
$$

is valid for any vector function $v \in \mathcal{W}_{\varangle}\left(\Omega_{\varangle}\right)$, where $r=|x|$ is the distance to the cusp tip $\mathcal{O}$ and the multiplier $K_{\varangle}$ depends on the sectorial domain $\Omega_{\varangle}$ only.

Proof First of all, we mention that, under the Dirichlet conditions from (5.13) on parts of the surfaces $\Sigma_{0}$ and $\Sigma_{\pi / 3}$ with positive area, any rigid motion, either a translation $c \in \mathbb{R}^{3}$, or rotation $c \times x$, becomes null. ${ }^{4}$

\footnotetext{
${ }^{4}$ It is not the case for the perpendicular planes $\Sigma_{0}$ and $\Sigma_{\pi / 2}$. To support the inference, the angle must be acute, for example, $\pi / N$ with $N=3,4,5, \ldots$, cf., [35].
} 
We divide the domain $\Omega$ into the subdomains

$$
\Omega \backslash \overline{\Pi_{d}} \quad \text { and } \quad \vartheta_{j}=\left\{x \in \Pi_{d}: z \in\left(d(1+j)^{-1}, d j^{-1}\right)\right\}, \quad j \in \mathbb{N} .
$$

Owing to the above observation on rigid motions, the Korn inequality is valid, that is,

$$
\left\|u ; L^{2}\left(\Omega \backslash \overline{\Pi_{d}}\right)\right\|^{2} \leq C\left\|D(\nabla) u ; L^{2}\left(\Omega \backslash \overline{\Pi_{d}}\right)\right\|^{2} .
$$

Diameters of the bases of the fragments $\vartheta_{j}$ are $O\left(j^{-2}\right)$ and its height $d j^{-1}-d(1+j)^{-1}=$ $d j^{-1}(1+j)^{-1}$ gets the same order as $j \rightarrow+\infty$. Moreover, the set $\vartheta_{j}$ is star-shaped with respect to a ball of radius $\varrho j^{-2}$ with a common for all $j$ constant $\varrho$. Thus, a result in [36] (see also [15, Ch. 2, §1]) assures the Korn inequality

$$
j^{-4}\left\|u ; L^{2}\left(\vartheta_{j}\right)\right\|^{2} \leq c\left\|\nabla u ; L^{2}\left(\vartheta_{j}\right)\right\|^{2} \leq C\left\|D(\nabla) u ; L^{2}\left(\vartheta_{j}\right)\right\|^{2} .
$$

Since $0<c j^{-1}<r<C j^{-1}$ for $x \in \vartheta_{j}$, we change $j^{-4}$ on the left of (5.15) for $r^{-2}$ inside the $L^{2}\left(\vartheta_{j}\right)$-norm of $u$. Summing all these inequalities gives (5.14).

An immediate consequence of the unbounded weight $r^{-2}$ on the left-hand side of (5.14) is that the energy space (5.13) belongs to the Sobolev space $H^{1}\left(\Omega_{\varangle}\right)^{3}$ and, moreover, is compactly embedded into the Lebesgue space $L^{2}\left(\Omega_{\varangle}\right)^{3}$. Thus, the spectrum of the problem (5.12) is fully discrete and constitutes a monotone unbounded sequence of eigenvalues

$$
0<\Lambda_{\varangle}^{(1)} \leq \Lambda_{\varangle}^{(2)} \leq \cdots \leq \Lambda_{\varangle}^{(k)} \leq \rightarrow+\infty
$$

where their multiplicity is taken into account. The corresponding eigenmodes $u_{\varangle}^{(k)} \in$ $\mathcal{W}_{\varangle}\left(\Omega_{\varangle}\right)$ can be subject to the normalization and orthogonality conditions

$$
\left(u_{\varangle}^{(k)}, u_{\varangle}^{(j)}\right)_{\Omega_{\varangle}}=\delta_{k, j}, \quad j, k \in \mathbb{N} .
$$

Proposition 3 Any eigenmode $u_{\varangle}^{(k)}$ in the problem (5.12) satisfies the inclusions $e^{-\delta / z} \nabla u_{\varangle}^{(k)} \in$ $L^{2}\left(\Omega_{\varangle}\right)$ and $z^{-2} e^{-\delta / z} u_{\varangle}^{(k)} \in L^{2}\left(\Omega_{\varangle}\right)$, where $\delta$ is a (small) positive number.

Proof We introduce a continuous piecewise smooth weighting function

$$
\mathcal{R}_{\tau, \delta}(z)= \begin{cases}e^{\delta / z} & z>\tau, \\ e^{\delta / \tau} & z \leq \tau\end{cases}
$$

where $\delta$ and $\tau$ are small positive, and insert into the integral identity (5.12) the test function $v=\mathcal{R}_{\delta, \tau}^{2} u_{\varangle}^{(k)}$ which falls into $\mathcal{W}_{\varangle}\left(\Omega_{\varangle}\right)$ together with $u$ because the weighting function (5.18) is constant near the cusp tip $\mathcal{O}$. Simple calculations transferring one factor $\mathcal{R}_{\delta, \tau}$ from $v$ onto $u_{\varangle}^{(k)}$ and commuting it twice with the differential operator (1.9) lead to the relation

$$
\begin{aligned}
& a\left(\mathcal{R}_{\delta, \tau} u_{\varangle}^{(k)}, \mathcal{R}_{\delta, \tau} u_{\varangle}^{(k)} ; \Omega_{\varangle}\right)-\left(A \mathcal{K}_{\delta, \tau} \mathcal{R}_{\delta, \tau} u_{\varangle}^{(k)}, \mathcal{K}_{\delta, \tau} \mathcal{R}_{\delta, \tau} u_{\varangle}^{(k)}\right)_{\Omega_{\varangle}} \\
& \quad=\Lambda_{\varangle}^{(k)}\left(\mathcal{R}_{\delta, \tau} u_{\varangle}^{(k)}, \mathcal{R}_{\delta, \tau} u_{\varangle}^{(k)}\right)_{\Omega_{\varangle}},
\end{aligned}
$$

where $\mathcal{K}_{\delta, \tau}=\mathcal{R}_{\delta, \tau}^{-1} D\left(\nabla_{x}\right) \mathcal{R}_{\delta, \tau}$ is a $6 \times 3$-matrix function such that

$$
\mathcal{K}_{\delta, \tau}(z) \leq \delta z^{-2} \text { and } \quad \mathcal{K}_{\delta, \tau}(z)=0 \text { for } z<\tau .
$$


Thus, the inequality (5.14) assures that

$$
\left(A \mathcal{K}_{\delta, \tau} \mathcal{R}_{\delta, \tau} u_{\varangle}^{(k)}, \mathcal{K}_{\delta, \tau} \mathcal{R}_{\delta, \tau} u_{\varangle}^{(k)}\right)_{\Omega_{\varangle}} \leq c \delta^{2} a\left(\mathcal{R}_{\delta, \tau} u_{\varangle}^{(k)}, \mathcal{R}_{\delta, \tau} u_{\varangle}^{(k)} ; \Omega_{\varangle}\right) .
$$

Recalling (5.17), we also write

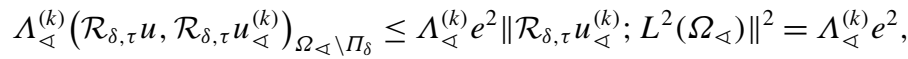

$$
\begin{aligned}
& \Lambda_{\varangle}^{(k)}\left(\mathcal{R}_{\delta, \tau} u_{\varangle}^{(k)}, \mathcal{R}_{\delta, \tau} u_{\varangle}^{(k)}\right)_{\Omega_{\varangle} \cap \Pi_{\delta}} \leq \Lambda_{\varangle}^{(k)} \delta^{-4}\left\|z^{-2} \mathcal{R}_{\delta, \tau} u_{\varangle}^{(k)} ; L^{2}\left(\Omega_{\varangle} \cap \Pi_{\delta}\right)\right\|^{2} .
\end{aligned}
$$

Now combining (5.19) and (5.20), (5.21) and choosing $\delta>0$ small yield

$$
\begin{aligned}
\frac{1}{2} a\left(\mathcal{R}_{\delta, \tau} u_{\varangle}^{(k)}, \mathcal{R}_{\delta, \tau} u_{\varangle}^{(k)} ; \Omega_{\varangle}\right) \leq & a\left(\mathcal{R}_{\delta, \tau} u_{\varangle}^{(k)}, \mathcal{R}_{\delta, \tau} u_{\varangle}^{(k)} ; \Omega_{\varangle}\right) \\
& -\left(A \mathcal{K}_{\delta, \tau} \mathcal{R}_{\delta, \tau} u_{\varangle}^{(k)}, \mathcal{K}_{\delta, \tau} \mathcal{R}_{\delta, \tau} u_{\varangle}^{(k)}\right)_{\Omega_{\varangle}} \\
& -\Lambda_{\varangle}^{(k)}\left(\mathcal{R}_{\delta, \tau} u_{\varangle}^{(k)}, \mathcal{R}_{\delta, \tau} u_{\varangle}^{(k)}\right)_{\Omega_{\varangle} \cap \Pi_{\delta}} \\
= & \Lambda_{\varangle}^{(k)}\left(\mathcal{R}_{\delta, \tau} u_{\varangle}^{(k)}, \mathcal{R}_{\delta, \tau} u_{\varangle}^{(k)}\right)_{\Omega_{\varangle} \backslash \Pi_{\delta}} \leq \Lambda_{\varangle}^{(k)} e^{2} .
\end{aligned}
$$

Since the right-hand side becomes uniformly bounded in $\tau \in(0, d)$, the left-hand side does the same and the limit passage $\tau \rightarrow+0$ assures that

$$
\left\|z^{-2} e^{\delta / z} u_{\varangle}^{(k)} ; L^{2}\left(\Omega_{\varangle}\right)\right\|^{2} \leq c\left\|e^{\delta / z} \nabla_{x} u_{\varangle}^{(k)} ; L^{2}\left(\Omega_{\varangle}\right)\right\|^{2} \leq C_{k} .
$$

The proposition is verified.

Proposition 3 proves that the eigenmodes $u_{\varangle}^{(k)}$ decay exponentially as $x \rightarrow \mathcal{O}$. Furthermore, the weighted Hölder estimates [37] show that, maybe with a smaller $\delta>0$,

$$
\left|u_{\varangle}^{(k)}(x)\right| \leq c e^{-\delta / z}, \quad\left|\varepsilon\left(u_{\varangle}^{(k)} ; x\right)\right| \leq C e^{-\delta / z} .
$$

The second distinguishing feature of the imposed artificial boundary conditions allows us to construct an eigenmode of the original problem (1.10), (1.11) in the intact domain $\Omega$ from any eigenmode $u_{\varangle}^{(k)}$ in the sectorial subdomain (5.9). Indeed, the odd for $\left(u_{\varangle}^{(k)}\right)_{n}$ and even for $\left(u_{\varangle}^{(k)}\right)_{s^{j}}$ extensions in the $n$-direction through the surface $\Sigma_{0}$ remain smooth and keep the differential equations (1.10) in $\{x \in \Omega: \varphi \in(-\pi / 3,0)\}$ and the boundary conditions (1.11) on $\{x \in \Gamma: \varphi \in(-\pi / 3,0)\}$ in view of the mirror symmetry accepted by homogeneous isotropic media. At the same time, the even for $\left(u_{\varangle}^{(k)}\right)_{n}$ and odd for $\left(u_{\varangle}^{(k)}\right)_{s^{j}}$ extensions in the $n$-direction through the surface $\Sigma_{\pi / 3}$ do the same with the differential equation (1.10) in the next sector $\{x \in \Omega: \varphi \in(\pi / 3,2 \pi / 3)\}$ and the boundary conditions (1.11) on the fragment $\{x \in \Gamma: \varphi \in(\pi / 3,2 \pi / 3)\}$ of the sector boundary. Repeating this two-fold extension procedure three times turns $u_{\varangle}^{(k)}$ into a smooth eigenmode in $\Omega$ corresponding to the same eigenvalue. In this way, the point spectrum $\wp_{p o}$ contains the whole infinite sequence (5.16). In $[1,35]$ some other shapes of the cross-section and orthotropic elastic materials are listed which also support the point spectrum of infinite total multiplicity in a cuspidal solid.

In the absence of the physical and geometrical symmetry assumed above to impose the special artificial boundary conditions (5.10), (5.11) and to conclude the existence of the unbounded sequence (5.16), embedded eigenvalues of course may emerge as well but in general the corresponding trapped modes lose the very fast decay rate (5.22) at the cusp tip $\mathcal{O}$ and obey relations of type (5.7), where $\kappa_{\min }(\Lambda)$ is again defined by (5.8). Recall that there 
are two positive roots in (3.9) for $\Lambda \in\left[\Lambda_{2}^{\dagger},+\infty\right)$ but three positive roots for $\Lambda \in\left[\Lambda_{1}^{\dagger}, \Lambda_{2}^{\dagger}\right.$ ). Hence, the number $\aleph(\Lambda)$ of energy power-law solutions is computed as follows:

$$
\begin{array}{ll}
\aleph(\Lambda)=6 & \text { for } \Lambda \in\left(0, \Lambda^{\dagger}\right), \\
\aleph(\Lambda)=5 & \text { for } \Lambda \in\left[\Lambda_{1}^{\dagger}, \Lambda_{2}^{\dagger}\right), \\
\aleph(\Lambda)=4 & \text { for } \Lambda \in\left[\Lambda_{2}^{\dagger},+\infty\right) .
\end{array}
$$

The space of trapped modes is further denoted by $\mathcal{L}_{\Lambda}$. It is always a finite-dimensional subspace and admits the decomposition

$$
\mathcal{L}_{\Lambda}=\mathcal{L}_{\Lambda}^{\text {exp }} \oplus \mathcal{L}_{\Lambda}^{\text {pow }}
$$

where $\mathcal{L}_{\Lambda}^{\text {exp }}$ consists of all trapped modes with the exponential decay (5.22) while $\operatorname{dim} \mathcal{L}_{\Lambda}^{\text {pow }} \leq \aleph(\Lambda)$

\subsection{The Augmented Scattering Matrix}

The change of variables (3.28) reduces the problem (1.10), (1.11) in the cusp (1.1) to a perturbed elasticity problem in the semi-infinite cylinder $Q_{d}=\varpi \times(-\infty, 1 / d)$. The Kondratiev theory [38] proves (or disproves for certain forbidden weight indexes) the Fredholm property of the corresponding operator in the weighted Sobolev spaces with the norms

$$
\left\|u ; W_{\beta}^{l}\left(Q_{d}\right)\right\|=\sum_{k=0}^{l}\left\|e^{-\beta t}\left|\nabla_{\eta, t}^{k} u\right| ; L^{2}\left(Q_{d}\right)\right\|
$$

which can be easily transformed into exponentially weighted Sobolev norms in the original domain $\Omega$. In this way, the operators $B_{\Lambda}( \pm \beta)$ with the domain $W_{\beta}^{2}(\Omega)$ become Fredholm if $\beta>0$ is small. However, for $\Lambda \in \wp_{c o}=\left[\Lambda^{\dagger},+\infty\right)$, the most interesting operator $B_{\Lambda}(0)$ with the null weight index $\beta$, which annihilates the exponential factor in (5.24) and turns (5.24) into the usual Sobolev norm, loses the Fredholm property due to the presence of waves (3.18) giving rise to singular Weyl sequences, see [1, 6, 7].

Let us compare the Fredholm operators $B_{\Lambda}^{-}=B_{\Lambda}(-\beta)$ and $B_{\Lambda}^{+}=B_{\Lambda}(+\beta)$ defined on vector functions with exponential decay and growth as $x \rightarrow \mathcal{O}$, respectively. These operators are formally adjoint to each other. According to the notation introduced above, the kernel of the operator $B_{\Lambda}^{-}$is composed from exponentially decaying trapped modes and takes the form

$$
\operatorname{ker} B_{\Lambda}^{-}=\mathcal{L}_{\Lambda}^{\text {exp }}
$$

At the same time, the cokernel of the adjoint operator $B_{\Lambda}^{+}$consists of the defect functionals (5.2) with $w \in \mathcal{L}_{\Lambda}^{\text {exp }}$.

Observing that $W_{\beta}^{2}(\Omega) \subset W_{-\beta}^{2}(\Omega)$, we are going to describe the component $\mathcal{Z}_{\Lambda}$ in the decomposition

$$
\operatorname{ker} B_{\Lambda}^{+}=\operatorname{ker} B_{\Lambda}^{-} \oplus \mathcal{Z}_{\Lambda} .
$$

Since the adjoint operator for $B_{\Lambda}^{ \pm}$is nothing but $B_{\Lambda}^{\mp}$, we have

$$
\operatorname{dim} \operatorname{ker} B_{\Lambda}^{ \pm}=\operatorname{dim} \operatorname{coker} B_{\Lambda}^{\mp} \text {. }
$$


Calculations in Sect. 3.2 displayed $12=4+4+2+2$ waves in the cusp (1.1) and the theorem on the index increment (cf., [14, Theorem 3.3.3]) assures that

$$
\operatorname{Ind} B_{\Lambda}^{+}=\operatorname{Ind} B_{\Lambda}^{-}+12,
$$

where $\operatorname{Ind} B=\operatorname{dim} \operatorname{ker} B-\operatorname{dim} \operatorname{coker} B$ is the index of a Fredholm operator $B$. Comparing (5.25)-(5.27), we conclude that

$$
\operatorname{Ind} B_{\Lambda}^{+}=-\operatorname{Ind} B_{\Lambda}^{-}=6, \quad \operatorname{dim} \mathcal{Z}_{\Lambda}=6 .
$$

Let us demonstrate some solutions $\mathfrak{Z}^{1}, \ldots, \mathfrak{Z}^{6}$ which form a basis in the subspace $\mathcal{Z}_{\Lambda}$. To this end, it is convenient to extend the vector/matrix notation and build the rows of incoming (plus) and outgoing (minus) waves and wave packets introduced in Sect. 4:

$$
u^{ \pm}=\left(u^{1 \pm}, \ldots, u^{6 \pm}\right)=\left(u_{\approx}^{ \pm}, u_{\bullet}^{ \pm}\right)
$$

Here, the rows $u_{\approx}^{ \pm}$of length $6-\aleph(\Lambda)$ are composed from oscillating waves and logarithmic packets, see Sects. 4.3 and 4.4 , while the rows

$$
u_{\bullet}^{ \pm}=\frac{1}{\sqrt{2}}\left(u_{\mathbf{\Delta}} \mp i u_{\mathbf{v}}\right)
$$

of length $\aleph(\Lambda)$ contain non-energy wave packets (4.16) constructed from all energy and non-energy waves defined in Sect. 4.5 and included into the rows

$$
u_{\mathbf{\Delta}}=\left(u^{10}, \ldots, u^{0 \aleph(\Lambda)}\right), \quad u_{\nabla}=\left(u^{11}, \ldots, u^{1 \aleph(\Lambda)}\right) .
$$

The achieved orthogonality and normalization conditions (4.11) in the matrix form read

$$
q_{0}\left(u^{ \pm}, u^{ \pm}\right)= \pm i \mathbb{I}_{6}, \quad q_{0}\left(u^{ \pm}, u^{\mp}\right)=\mathbb{O}_{6},
$$

where $\mathbb{I}_{N}$ and $\mathbb{O}_{N}$ are the unit and null matrices of size $N \times N$, respectively. Moreover, the equality (4.12) and similar relations for the packets (4.15) and (4.16) guarantee that

$$
u_{\approx}^{+}(x)=\overline{u_{\approx}^{-}(x)}, \quad u_{\diamond}^{+}(x)=\overline{u_{\diamond}^{-}(x)}
$$

Proposition 4 For any $\Lambda>0$, there exists the row $\mathfrak{Z}=\left(\mathfrak{Z}^{1}, \ldots, \mathfrak{Z}^{6}\right)$ of solutions to the problem (1.10), (1.11) in the asymptotic form

$$
\mathfrak{Z}(x)=\chi(z) u^{+}(x)+\chi(z) u^{-}(x) \mathfrak{S}+\widetilde{\mathfrak{Z}}(x),
$$

where $\chi$ is a smooth cut-off function such that

$$
\chi(z)=1 \text { for } z<d / 2 \text { and } \chi(z)=0 \text { for } z>d,
$$

the remainder $\widetilde{\mathfrak{Z}}(x)$ decays exponentially as $x \rightarrow \mathcal{O}$, and the $6 \times 6$-matrix $\mathfrak{S}$ is unitary and symmetric. The solutions $\mathfrak{Z}^{1}, \ldots, \mathfrak{Z}^{6}$ constitute a basis in the subspace $\mathcal{Z}_{\Lambda}$.

Proof Any non-trivial element $\mathfrak{z}$ of the subspace $\mathcal{L}_{\Lambda}$ admits the representation

$$
\mathfrak{z}=\chi u^{+} \mathfrak{c}^{+}+\chi u^{-} \mathfrak{c}^{-}+\tilde{\mathfrak{z}}
$$


with an exponentially decaying remainder $\tilde{\mathfrak{z}}$ and some coefficient column vectors $\mathfrak{c}^{ \pm} \in \mathbb{C}^{6}$ such that $\left|\mathfrak{c}^{+}\right|+\left|\mathfrak{c}^{-}\right| \neq 0$ (otherwise, $\mathfrak{z} \in \mathcal{L}_{\Lambda}^{\text {exp }}$ ). Let us assume that $\mathfrak{c}^{+}=0$. Inserting $\mathfrak{z}$ into Green's formula in the truncated domain $\Omega_{\delta}=\Omega \backslash \overline{\Pi_{\delta}}$, we compute the limit, cf., (4.6),

$$
0=\lim _{\delta \rightarrow+0} q_{\delta}(\mathfrak{z}, \mathfrak{z})=\lim _{\delta \rightarrow+0} q_{\delta}\left(u^{-} \mathfrak{c}^{-}, u^{-} \mathfrak{c}^{-}\right)=-i\left|\mathfrak{c}^{-}\right|^{2}
$$

and come across a contradiction. Since the incoming waves $u^{1+}, \ldots, u^{6+}$ are linearly independent and $\operatorname{dim} \mathcal{Z}_{\Lambda}=6$, the existence of the solutions $\mathfrak{Z}^{1}, \ldots, \mathfrak{Z}^{6}$ forming a basis in $\mathcal{Z}_{\Lambda}$ and composing the row (5.33) has become clear.

It suffices to verify the properties of the matrix $\mathfrak{S}$. To this end, we first apply the orthogonality and normalization conditions (5.32) and repeat the calculation (5.34) in the matrix form

$$
0=\lim _{\delta \rightarrow+0} q_{\delta}(\mathfrak{Z}, \mathfrak{Z})=\lim _{\delta \rightarrow+0} q_{\delta}\left(u^{+}+u^{-} \mathfrak{S}, u^{+}+u^{-} \mathfrak{S}\right)=i \mathbb{I}_{6}-i \mathfrak{S}^{*} \mathfrak{S} .
$$

This equality with the adjoint matrix $\mathfrak{S}^{*}$ establishes the unitary property. Second, we write

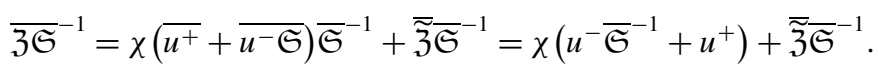

The representation of this row of solutions has the same incoming waves $u^{+}$as the original row $\mathfrak{Z}$. Thus according to a consequence of our calculation (5.34) the difference $\mathfrak{Z}-\overline{\mathfrak{Z}}$ gets the exponential decay as $x \rightarrow \mathcal{O}$ and we obtain

$$
\mathfrak{S}=\overline{\mathfrak{S}}^{-1}=\overline{\mathfrak{S}}^{*}=\mathfrak{S}^{\top}
$$

The proof is completed.

We call $\mathfrak{S}$ in (5.33) the augmented scattering matrix and will use it in the next section.

\subsection{The Scattering Matrix and a Criterion for the Existence of Power-Law Trapped Modes}

According to the introduced decomposition of waves in (5.29), we rewrite the representation (5.33) of the row $\mathfrak{Z}=(\mathfrak{Z} \approx, \mathfrak{Z})$ in two lines

$$
\begin{aligned}
& \mathfrak{Z} \approx=\chi u_{\approx}^{+}+u_{\approx}^{-} \mathfrak{S} \approx \approx+u_{\bullet}^{-} \mathfrak{S} \approx+\widetilde{\mathfrak{Z}} \approx, \\
& \mathfrak{Z} \bullet=\chi u_{\downarrow}^{+}+u_{\approx}^{-} \mathfrak{S}_{\approx}+u_{\bullet}^{-} \mathfrak{S} \downarrow+\widetilde{\mathfrak{Z}}
\end{aligned}
$$

which involve blocks of the augmented scattering matrix

$$
\mathfrak{S}=\left(\begin{array}{ll}
\mathfrak{S}_{\approx} & \mathfrak{S}_{\approx} \\
\mathfrak{S} \approx & \mathfrak{S}_{\bullet}
\end{array}\right)
$$

Theorem 2 The component $\mathcal{L}_{\Lambda}^{\text {pow }}$ of the decomposition (5.23) satisfies the relation

$$
\operatorname{dim} \mathcal{L}_{\Lambda}^{\text {pow }}=\operatorname{dim} \operatorname{ker}\left(\mathfrak{S}_{\diamond}-\mathbb{I}_{\aleph(\Lambda)}\right),
$$

where the right-hand side is nothing but the multiplicity of the eigenvalue 1 of the right below block of the augmented scattering matrix (5.37). 
Proof Any nontrivial element $u \in \mathcal{L}_{\Lambda}^{\text {pow }}$ admits the representation

$$
\begin{aligned}
u=\mathfrak{Z} c= & \chi u_{\approx}^{+} c_{\approx}+\chi u_{\approx}^{-}\left(\mathfrak{S}_{\approx \approx} c_{\approx}+\mathfrak{S}_{\approx} c_{\diamond}\right) \\
& +\chi u_{\downarrow}^{+} c_{\diamond}+\chi u_{\diamond}^{-}\left(\mathfrak{S}_{\diamond} c_{\approx}+\mathfrak{S}_{\diamond} c_{\diamond}\right)+\widetilde{\mathfrak{Z}} c,
\end{aligned}
$$

where $c=\left(c_{\approx}, c_{\diamond}\right) \in \mathbb{C}^{6} \backslash\{0\}$ and the formulas (5.36) were taken into account. Since the oscillatory waves $u_{\approx}^{ \pm}$must be absent in (5.38), we obtain

$$
c_{\approx}=\mathfrak{S}_{\approx} c_{\diamond}=0 \in \mathbb{C}^{6-\aleph(\Lambda)}
$$

Hence, using (5.30) leads to

$$
u=\chi \frac{i}{\sqrt{2}} u_{\nabla}\left(\mathfrak{S}_{\diamond}-\mathbb{I}_{\aleph(\Lambda)}\right) c_{\diamond}+\chi \frac{1}{\sqrt{2}} u_{\mathbf{\Delta}}\left(\mathfrak{S}_{\diamond}+\mathbb{I}_{\aleph(\Lambda)}\right) c_{\diamond}+\tilde{\mathfrak{Z}}_{\downarrow} c_{\diamond}
$$

The non-energy waves composing the second row in (5.31) also have to disappear from the decomposition (5.39) of the trapped mode $u$ and, therefore,

$$
\mathfrak{S}_{\diamond} c_{\diamond}=c_{\diamond}
$$

At the same time, the coefficient column vector $2^{-1 / 2}\left(\mathfrak{S}_{\diamond}+\mathbb{I}_{\aleph(\Lambda)}\right) c_{\diamond}=2^{1 / 2} c_{\diamond}$ of the energy waves row $u_{\boldsymbol{\Delta}}$ does not vanish. In other words, any trapped mode in $\mathcal{L}_{\Lambda}^{\text {pow }} \backslash\{0\}$ gives rise to an eigenvector $c \_\operatorname{dim} \operatorname{ker}\left(\mathfrak{S}_{\downarrow}-\mathbb{I}_{\aleph(\Lambda)}\right) \backslash\{0\}$.

Let now $c_{\diamond} \in \operatorname{ker}\left(\mathfrak{S}_{\diamond}-\mathbb{I}_{\aleph(\Lambda)}\right)$. We observe that the column vector $c=\left(c_{\approx}, c_{\diamond}\right)$ with $c_{\approx}=0 \in \mathbb{C}^{6-\aleph(\Lambda)}$ falls into $\operatorname{ker}\left(\mathfrak{S}-\mathbb{I}_{6}\right)$ because the unitary property of the augmented scattering matrix assures that

$$
\left|c_{\Downarrow}\right|^{2}=|c|^{2}=\left|S_{c}\right|^{2}=\left|\mathfrak{S}_{\approx} c_{\Downarrow}\right|^{2}+\left|\mathfrak{S}_{\Downarrow} c_{\Downarrow}\right|^{2}=\left|\mathfrak{S}_{\approx} c_{\Downarrow}\right|^{2}+\left|c_{\Downarrow}\right|^{2} \quad \Rightarrow \quad \mathfrak{S}_{\approx} c_{\Downarrow}=0 .
$$

As a result, we see that the vector function $u=\mathfrak{Z} c=\mathfrak{Z} \triangleleft c$ takes the form (5.39) and, moreover, $u=\chi 2^{1 / 2} u_{\Delta} c_{\diamond}+\widetilde{\mathfrak{Z}} c_{\diamond} \in \mathcal{L}_{\Lambda}^{\text {pow }}$ in the case $c \diamond \neq 0$.

The proof is completed.

The relation (5.40) has an important inference, namely the $(6-\aleph(\Lambda)) \times(6-\aleph(\Lambda))$ matrix

$$
S=\mathfrak{S}_{\approx \approx}-\mathfrak{S}_{\approx}\left(\mathfrak{S}_{\diamond}-\mathbb{I}_{\aleph(\Lambda)}\right)^{-1} \mathfrak{S}_{\diamond}
$$

is well-defined and inherits the unitary and symmetry properties from the matrix $\mathfrak{S}$, cf., the matrix (3.4). The relationship (5.41) is verified by a direct calculation while, since $\mathfrak{S} \approx=$ $\mathfrak{S}_{\approx}^{\top}$, we have $\mathfrak{S}_{\approx} \approx c \in \operatorname{ker}\left(\mathfrak{S}_{\diamond}-\mathbb{I}_{\mathcal{N}(\Lambda)}\right)$ for any $c \in \mathbb{C}^{6}$ due to the implication (5.40) which also compensates for arbitrariness in the choice of the inverse $\left(\mathfrak{S}_{\downarrow}-\mathbb{I}_{\aleph(\Lambda)}\right)^{-1}$ on the range $\operatorname{Im} \mathfrak{S}_{\diamond}-\mathbb{I}_{\aleph(\Lambda)}$.

The matrix (5.41) gets registered as the scattering matrix in the problem (1.10), (1.11) because algebraic operations on its solutions (5.36) provide the new row of solutions

$$
\begin{aligned}
Z & =\mathfrak{Z} \approx \approx-\mathfrak{Z} \approx\left(\mathfrak{S} \downarrow-\mathbb{I}_{\aleph(\Lambda)}\right)^{-1} \mathfrak{S} \approx \\
& =\chi u_{\approx}^{+}+\chi u_{\approx}^{-} S+\chi 2^{1 / 2} u_{\Lambda}\left(\mathfrak{S} \downarrow-\mathbb{I}_{\aleph_{(\Lambda)}}\right)^{-1} \mathfrak{S} \approx+\widetilde{Z} .
\end{aligned}
$$

We rewrite (5.42) in the form

$$
Z=\left(Z^{1}, \ldots, Z^{6-\aleph(\Lambda)}\right)=\chi u_{\approx}^{+}+\chi u_{\approx}^{-} S+\widehat{Z}
$$


and observe that the new remainder falls into the energy space $\mathcal{W}(\Omega)$ because differs from the exponentially decaying vector function $\widetilde{Z}$ by a linear combination of the energy waves $u_{\mathbf{\Delta}}=\left(u^{10}, \ldots, u^{0 \aleph(\Lambda)}\right)$ only. In other words, the displacement fields $Z^{p}$ are initiated by the incoming waves $u^{p+}$ and the matrix $S$ describes the scattered field as a linear combination of the outgoing waves $u^{-}=\left(u^{1-}, \ldots, u^{6-\aleph(\Lambda)-}\right)$ with the reflection coefficients $S_{p 1}, \ldots, S_{p 6-\aleph(\Lambda)}$.

It is worth to mentioning that the augmented scattering matrix $\mathfrak{S}$ in Proposition 4 generates the traditional scattering matrix $S$ as well as the condition $\operatorname{dim} \operatorname{ker}\left(\mathfrak{S} \leftrightarrow-\mathbb{I}_{\aleph_{(\Lambda)}}\right)>0$ in Theorem 2 for the existence of trapped modes with a power-law behavior at the cusp tip $\mathcal{O}$ rejecting the exponential decay rate as was in Sect. 5.2. In this way, the augmented scattering matrix keeps a lot of information on the solvability of the elasticity problem in the cuspidal solid $\Omega$, although it is an artificial object.

\subsection{The Mandelstam (Energy) Radiation Conditions and the Solvability of the Elasticity Problem Above the Threshold}

We again consider the operators

$$
B_{\Lambda}^{ \pm}=B_{\Lambda}( \pm \beta): \mathcal{D}_{ \pm \beta}(\Omega) \rightarrow \mathcal{R}_{ \pm \beta}(\Omega)
$$

from Sect. 5.3 with their domains $\mathcal{D}_{-\beta}(\Omega)$ and $\mathcal{D}_{+\beta}(\Omega)$ and the ranges $\mathcal{R}_{-\beta}(\Omega)$ and $\mathcal{R}_{+\beta}(\Omega)$, respectively, consisting of vector functions with the exponential decay and possible exponential growth as $x \rightarrow \mathcal{O}$. The theorem on asymptotics in [7], see also Theorem 1, shows that a solution $u \in \mathcal{D}_{+\beta}(\Omega)$ of the problem (1.17), (1.18) with the right-hand sides $(f, g) \in \mathcal{R}_{-\beta}(\Omega)$ subject to the compatibility conditions

$$
(f, v)_{\Omega}+(g, v)_{\Gamma}=0 \quad \forall v \in \mathcal{L}_{\Lambda}^{\exp },
$$

cf., (5.2) and (5.4), admits the asymptotic form

$$
u=\chi u^{+} c^{+}+\chi u^{-} c^{-}+\tilde{u}
$$

with the remainder $\tilde{u} \in \mathcal{D}_{-\beta}(\Omega)$ and some coefficient column vectors $c^{ \pm} \in \mathbb{C}^{6}$. Using the special solutions (5.33), we find that the linear combination

$$
u^{a r t}=u-\mathfrak{Z}^{+}=\chi u^{-} c^{a r t}+\widetilde{u}^{a r t}
$$

involves only the outgoing waves $u^{-}=\left(u^{1-}, \ldots, u^{6-}\right)$ and satisfies the artificial Mandelstam radiation conditions because according to Sect. 4 it transports energy towards the cusp tip. The adjective "artificial" is added due to the appearance in (5.46) non-energy wave packets.

We compose the weighted space $\mathcal{D}_{\beta}^{\text {art }}(\Omega)$ with detached asymptotics from vector functions (5.46) with the norm

$$
\left\|u^{\text {art }} ; \mathcal{D}_{\beta}^{\text {art }}(\Omega)\right\|=\left|c^{\text {art }}\right|+\left\|\tilde{u}^{\text {art }} ; \mathcal{D}_{-\beta}(\Omega)\right\| .
$$

The restriction $B_{\Lambda}^{\text {art }}(\beta)$ of the operator (5.43) with the positive weight exponent $+\beta$ onto the subspace $\mathcal{D}_{\beta}^{\text {art }}(\Omega)$ becomes an operator of the problem (1.17), (1.18) with the artificial radiation conditions. 
Proposition 5 The operator $B_{\Lambda}^{\text {art }}(\beta)$ is Fredholm of index zero, that is, the problem (1.17), (1.18) with the right-hand sides $(f, g) \in \mathcal{R}_{-\beta}(\Omega)$ enjoying the compatibility conditions (5.44), has a solution (5.46) in the space $\mathcal{D}_{\beta}^{\text {art }}(\Omega)$ which is defined up to an addendum in $\mathcal{L}_{\Lambda}^{\text {exp }}$, an exponentially decaying trapped mode. Moreover, under the orthogonality conditions

$$
\left(u^{\text {art }}, v\right)_{\Omega}=0 \quad \forall v \in \mathcal{L}_{\Lambda}^{\text {exp }},
$$

the solution becomes unique and meets the estimate

$$
\left\|u^{\text {art }} ; \mathcal{D}_{\beta}^{\text {art }}(\Omega)\right\| \leq c\left\|(f, g) ; \mathcal{R}_{-\beta}(\Omega)\right\| .
$$

Proof The operator

$$
B_{\Lambda}^{\text {art }}(\beta): \mathcal{D}_{\beta}^{\text {art }}(\Omega) \rightarrow \mathcal{R}_{-\beta}(\Omega)
$$

is Fredholm since its domain differs from the domain $\mathcal{D}_{-\beta}(\Omega)$ of the Fredholm operator $B_{\Lambda}(-\beta)$ by a subspace of finite dimension 6 while both act into the same space $\mathcal{R}_{-\beta}(\Omega)$ of exponentially decaying right-hand sides. Moreover, by the relationship (5.28), its index equals

$$
\operatorname{Ind} B_{\Lambda}^{a r t}(\beta)=6+\operatorname{Ind} B_{\Lambda}(-\beta)=0 .
$$

The compatibility conditions (5.44) provide the existence of a solution in $\mathcal{D}_{+\beta}(\Omega)$ and the asymptotic representation verified in Theorem 1 gives it the form (5.45) while a simple transformation puts the desired solution (5.46) in the space $\mathcal{D}_{\beta}^{\text {art }}(\Omega)$. In other words, $\operatorname{dim} \operatorname{coker} B_{\Lambda}^{\text {art }}(\beta)=\operatorname{dim} \mathcal{L}_{\Lambda}^{\text {exp }}$. It remains to present just $\operatorname{dim} \mathcal{L}_{\Lambda}^{\text {exp }}$ linear independent solutions of the homogeneous problem in $\mathcal{D}_{+\beta}(\Omega)$, that is, any basis of trapped modes.

Proposition 6 Under the hypotheses of Proposition 5, the coefficient column vector $c^{\text {art }} \in$ $\mathbb{C}^{6}$ in (5.46) takes the form

$$
c^{\text {art }}=i \mathfrak{S}\left((f, \mathfrak{Z})_{\Omega}+(g, \mathfrak{Z})_{\partial \Omega}\right) .
$$

Proof Inserting $u^{\text {art }}$ and $\mathfrak{Z}$ into the truncated domain $\Omega_{\delta}$, we repeat our previous calculations (5.34) and (5.35). As a result, we obtain

$$
(f, \mathfrak{Z})_{\Omega}+(g, \mathfrak{Z})_{\partial \Omega}=\lim _{\delta \rightarrow+0} q_{\delta}\left(u^{\text {art }}, \mathfrak{Z}\right)=-i \mathfrak{S}^{*} c^{\text {art }}
$$

In the main assertion of our paper we, in particular, will get rid of non-energy waves in the representation of the artificial solution

$$
u^{a r t}=\chi u_{\approx}^{-} c_{\approx}^{a r t}+\chi u_{\diamond}^{-} c_{\diamond}^{a r t}+\widetilde{u}^{a r t}
$$

to achieve the desired solution accepting the Mandelstam energy radiation conditions.

Theorem 3 Let $(f, g) \in \mathcal{R}_{-\beta}(\Omega)$ satisfy the compatibility conditions (5.2), where $\mathcal{L}_{\Lambda}$ is the whole space (5.23) of trapped modes. Then problem (1.17), (1.18) has a solution in the form

$$
u^{\text {out }}=\chi u_{\approx}^{-} c_{\approx}^{\text {out }}+\chi u_{\mathbf{\Delta}}^{-} c_{\mathbf{\Delta}}^{\text {out }}+\widetilde{u}^{\text {out }},
$$


where the remainder $\tilde{u}^{\text {out }}$ falls into the space $\mathcal{D}_{-\beta}(\Omega)$, i.e., decays exponentially, and $c_{\approx}^{\text {out }} \in$ $\mathbb{C}^{6-\aleph(\Lambda)}, c_{\boldsymbol{\Lambda}}^{\text {out }} \in \mathbb{C}^{\aleph(\Lambda)}$. The solution $(5.48)$ is defined up to an addendum in $\mathcal{L}_{\Lambda}$, a trapped mode, but under the orthogonality conditions (5.3) becomes unique and meets the estimate

$$
\left|c_{\approx}^{\text {out }}\right|+\left|c_{\boldsymbol{\Lambda}}^{\text {out }}\right|+\left\|\tilde{u}^{\text {out }} ; \mathcal{D}_{-\beta}(\Omega)\right\| \leq c\left\|(f, g) ; \mathcal{R}_{-\beta}(\Omega)\right\| .
$$

Proof The equalities (5.2), in particular, support the compatibility conditions (5.44) and Proposition 5 give us a solution (5.47) and we search for solution (5.48) in the form

$$
u^{\text {out }}=u^{\text {art }}+\mathfrak{Z} \bullet b \vee \quad \text { with } c \diamond \in \mathbb{C}^{\aleph(\Lambda)} .
$$

In view of (5.36), (5.47) and (5.30) we have

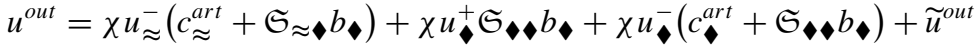

$$
\begin{aligned}
& =\chi u_{\approx}^{-}\left(c_{\approx}^{a r t}+\mathfrak{S}_{\approx} b_{\diamond}\right)+\chi \frac{i}{\sqrt{2}} u_{\nabla}\left(b_{\diamond}-\mathfrak{S}_{\diamond} b_{\diamond}-c_{\diamond}^{a r t}\right) \\
& +\chi \frac{i}{\sqrt{2}} u_{\boldsymbol{\Lambda}}\left(b \bullet+\mathfrak{S}_{\diamond} b+c_{\Downarrow}^{\text {art }}\right)+\tilde{u}^{\text {out }}, \quad \tilde{u}^{\text {out }} \in \mathcal{D}_{-\beta}(\Omega) .
\end{aligned}
$$

Our immediate objective becomes to find a coefficient column vector $b$ such that

$$
b \bullet-\mathfrak{S}_{\diamond} b b_{\diamond}^{a r t}
$$

To this end, we only need to assure that

$$
\bar{a}^{\top} b_{\diamond}=0 \quad \forall a \bullet \in \operatorname{ker}\left(\mathfrak{S}_{\diamond}-\mathbb{I}_{\aleph(\Lambda)}\right) .
$$

Let $a=\left(a_{\approx}, a_{\triangleleft}\right)$ with $a_{\approx}=0$. Owing to Proposition 6 , we have

$$
\begin{aligned}
\left(f, \mathfrak{Z} a_{\diamond}\right)_{\Omega}+(g, \mathfrak{Z} \triangleleft a \diamond)_{\partial \Omega} & =(f, \mathfrak{Z} a)_{\Omega}+(g, \mathfrak{Z} a)_{\partial \Omega}=\bar{a}^{\top}\left((f, \mathfrak{Z})_{\Omega}+(g, \mathfrak{Z})_{\partial \Omega}\right) \\
& =-i \bar{a}^{\top} \mathfrak{S}^{*} c^{a r t}=-i \overline{\mathfrak{S} a}^{\top} c^{a r t} \\
& =-i{\overline{\mathfrak{S}} \diamond a a^{\top}}^{\top} c^{a r t}=-i \bar{a}^{\top} c^{\text {art }} .
\end{aligned}
$$

Here, we have used the implication (5.40). Furthermore, in the proof of Theorem 2 we have concluded that $\mathfrak{Z}_{\downarrow} a_{\diamond} \in \mathcal{L}_{\Lambda}^{\text {pow }}$ in the situation (5.51) so that the expression (5.52) vanishes due to the assumed compatibility conditions (5.2). Thus, the necessary column vector $b$ indeed exists and is defined up to an addendum in $\operatorname{ker}\left(\mathfrak{S}_{\downarrow}-\mathbb{I}_{\aleph(\Lambda)}\right)$. In other words, (5.50) is satisfied, the non-energy waves disappear from (5.51), and solution (5.49) takes the form (5.48) being defined up to an addendum in $\mathcal{L}_{\Lambda}$. Other assertions either follow from Proposition 5 , or are evident.

Acknowledgements This work was written within the project 17-11-01003 of Russian Science Foundation. The authors are grateful to the anonymous referee whose comments helped to improve the presentation.

Open Access This article is distributed under the terms of the Creative Commons Attribution 4.0 International License (http://creativecommons.org/licenses/by/4.0/), which permits unrestricted use, distribution, and reproduction in any medium, provided you give appropriate credit to the original author(s) and the source, provide a link to the Creative Commons license, and indicate if changes were made. 


\section{References}

1. Nazarov, S.A.: The spectrum of the elasticity problem for a spiked body. Sib. Mat. Zh. 49(5), 1105-1127 (2008) (English transl.: Sib. Math. J. 49(5), 874-893 (2008))

2. Mironov, M.A.: Propagation of a flexural wave in a plate whose thickness decreases smoothly to zero in a finite interval. Sov. Phys. Acoust. 34(3), 318-319 (1988)

3. Mironov, M.A.: Exact solutions to equations of transverse oscillations of a rod with a special law of varying the cross-section along its axis. In: Proc. the XIth All-Union Acoustic Conference. Section L. Acoust. Inst. M. (1991) (in Russian)

4. Krylov, V.V.: New type of vibration dampers utilising the effect of acoustic "black holes". Acta Acust. Acust. 90(5), 830-837 (2004)

5. Krylov, V.V., Tilman, F.J.B.S.: Acoustic "black holes" for flexural waves as effective vibration dampers. J. Sound Vib. 274, 605-619 (2004)

6. Bakharev, F.L., Nazarov, S.A.: On the structure of the spectrum of the elasticity problem for a body with a super-sharp spike. Sib. Mat. Zh. 50(4), 746-756 (2009) (English transl.: Sib. Math. J. 50(4), 587-595 (2009))

7. Kozlov, V., Nazarov, S.A.: On the spectrum of an elastic solid with cusps. Adv. Differ. Equ. 21(9/10), 887-944 (2016)

8. Kozlov, V., Maz'ya, V.: Differential Equations with Operator Coefficients (with Applications to Boundary Value Problems for Partial Differential Equations). Monographs in Mathematics. Springer, Berlin (1999)

9. Kozlov, V., Maz'ya, V.: An asymptotic theory of higher-order operator differential equations with nonsmooth nonlinearities. J. Funct. Anal. 217, 448-488 (2004)

10. Nazarov, S.A.: Notes to the proof of a weighted Korn inequality for an elastic body with peak-shaped cusps. Probl. Mat. Anal. 63, 83-113 (2012) (English transl.: J. Math. Sci. 181(5), 632-667 (2012))

11. Lurie, A.I.: Theory of Elasticity. Springer, Berlin (2005)

12. Truesdell, C. (ed.): Linear theories of elasticity and thermoelasticity; linear and nonlinear theories of rods, plates, and shells. In: Mechanics of Solids, II. Springer, Berlin (1984). Reprint of the 1972 original

13. Sanchez-Hubert, J., Sanchez Palencia, E.: Statics of curved rods on account of torsion and flexion. Eur. J. Mech. A, Solids 18(3), 365-390 (1999)

14. Nazarov, S.A., Plamenevsky, B.A.: Elliptic Problems in Domains with Piecewise Smooth Boundaries. de Gruyter, Berlin (1994)

15. Nazarov, S.A.: Asymptotic Theory of Thin Plates and Rods, vol. 1. Dimension Reduction and Integral Estimates. Nauchnaya Kniga (IDMI), Novosibirsk (2002) (in Russian)

16. Panasenko, G.P.: Multi-Scale Modelling for Structures and Composites. Springer, Dordrecht (2005)

17. Nazarov, S.A.: Justification of the asymptotic theory of thin rods. Integral and pointwise estimates. Probl. Mat. Anal. 17, 101-152 (1997) (English transl.: J. Math. Sci. 97(4), 4245-4279 (1999))

18. Nazarov, S.A., Polyakova, O.R.: Asymptotic behavior of the stress-strain state near a spatial singularity of the boundary of the beak tip type. Prikl. Mat. Meh. 57(5), 130-149 (1993) (English transl.: J. Appl. Math. Mech. 57(5), 887-902 (1993))

19. Nazarov, S.A., Slutskii, A.S.: The asymptotic form of the stressed state near a three-dimensional boundary singularity of the "claw" type. Prikl. Mat. Meh. 63(6), 1008-1017 (1999). (English transl: J. Appl. Math. Mech. 63(6), 943-951 (1999))

20. Cardone, G., Nazarov, S.A., Taskinen, J.: A criterion for the existence of the essential spectrum for beak-shaped elastic bodies. J. Math. Pures Appl. 92(6), 628-650 (2009)

21. Nazarov, S.A.: Korn's inequalities for elastic junctions of massive bodies and thin plates and rods. Usp. Mat. Nauk 63(1), 37-110 (2008) (English transl.: Russ. Math. Surv. 63(1), 35-107 (2008))

22. Nazarov, S.A., Slutskii, A.S.: One-dimensional equations of deformation of thin slightly curved rods. Asymptotical analysis and justification. Izv. Akad. Nauk Ser. Mat. 64(3), 97-130 (2000) (English transl.: Math. Izv. 64(3), 531-562 (2000))

23. Kozlov, V.A., Maz'ya, V.G., Movchan, A.B.: Asymptotic Analysis of Fields in Multi-Structures. Oxford Math. Monogr. Clarendon Press, Oxford (1999)

24. Kuznetsov, N., Porter, R., Evans, D.V., Simon, M.J.: Uniqueness and trapped modes for surface-piercing cylinders in oblique waves. J. Fluid Mech. 365, 351-368 (1998)

25. Birman, M.S., Solomyak, M.Z.: Spectral Theory and Self-Adjoint Operators in Hilbert Space (in Russian). Leningr. Univ. Press, Leningrad (1980) (English transl.: Reidel, Dordrecht (1987))

26. Bouche, D., Molinet, F., Mittra, R.: Asymptotic Methods in Electromagnetics. Springer, Berlin (1997). $526 \mathrm{pp}$.

27. Wilcox, C.H.: Scattering Theory for Diffraction Gratings. Applied Mathematical Sciences Series, vol. 46. Springer, Berlin (1997). 525 pp. 
28. Mindlin, R.D.: Waves and Vibration in Isotropic Elastic Plate. Structural Mechanics, Proceedings of the 1st Symposium of Naval Structural Mechanics. Pergamon, Elmsford (1960)

29. Vorovich, I.I., Dynamical, B.V.A.: Mixed Problems of Elasticity for Nonclassical Domains. Nauka, Moscow (1979) (in Russian)

30. Nazarov, S.A.: Umov-Mandel'stam radiation conditions in elastic periodic waveguide. Mat. Sb. 205(7), 43-72 (2014) (English transl.: Sb. Math. 205(7), 953-982 (2014))

31. Umov, N.A.: Equations of Energy Motion in Bodies (in Russian). Odessa (1874)

32. Poynting, J.H.: On the transfer of energy in the electromagnetic field. Philos. Trans. R. Soc. Lond. 175, 343-361 (1884)

33. Mandelstam, L.I.: Lectures on Optics, Relativity, and Quantum Mechanics, vol. 2. Izv. AN SSSR, Moscow (1947)

34. Nazarov, S.A., Plamenevskii, B.A.: On radiation conditions for selfadjoint elliptic problems. Dokl. Akad. Nauk SSSR 311(3), 532-536 (1990) (English transl.: Sov. Math. Dokl. 41(2), 274-277 (1990))

35. Nazarov, S.A.: Trapped modes for a cylindrical elastic waveguide with a damping gasket. Ž. Vyčisl. Mat. Mat. Fiz. 48(5), 863-881 (2008) (English transl.: Comput. Math. Math. Phys. 48(5), 863-881 (2008))

36. Kondrat'ev, V.A., Olejnik, O.A.: Boundary value problems for the system of elasticity theory in unboundend domains. Korn's inequalities. Russ. Math. Surv. 43, 65-119 (1988)

37. Maz'ya, V.G., Plamenevskii, B.A.: Estimates in $L_{p}$ and in Hölder classes, and the Miranda-Agmon maximum principle for solutions of elliptic boundary value problems in domains with singular points on the boundary. Math. Nachr. 81, 2582 (1978) (English transl.: Am. Math. Soc. Transl. 123, 156 (1984))

38. Kondrat'ev, V.A.: Boundary value problems for elliptic equations in domains with conical or angular points. Tr. Mosk. Mat. Obŝ. 16, 209-292 (1967) (English transl.: Trans. Mosc. Math. Soc. 16, 227-313 (1967)) 